\title{
Viscous and Resistive Effects on the MRI with a Net Toroidal Field
}

\author{
Jacob B. Simon, John F. Hawley \\ Department of Astronomy \\ University of Virginia \\ P.O. Box 400325 \\ Charlottesville, VA 22904-4325
}

\begin{abstract}
Resistivity and viscosity have a significant role in establishing the energy levels in turbulence driven by the magnetorotational instability (MRI) in local astrophysical disk models. This study uses the Athena code to characterize the effects of a constant shear viscosity $\nu$ and Ohmic resistivity $\eta$ in unstratified shearing box simulations with a net toroidal magnetic flux. A previous study of shearing boxes with zero net magnetic field performed with the ZEUS code found that turbulence dies out for values of the magnetic Prandtl number, $P_{\mathrm{m}}=\nu / \eta$, below $P_{\mathrm{m}} \sim 1$; for $P_{\mathrm{m}} \gtrsim 1$, time- and volume-averaged stress levels increase with $P_{\mathrm{m}}$. We repeat these experiments with Athena and obtain consistent results. Next, the influence of viscosity and resistivity on the toroidal field MRI is investigated both for linear growth and for fully-developed turbulence. In the linear regime, a sufficiently large $\nu$ or $\eta$ can prevent MRI growth; $P_{\mathrm{m}}$ itself has little direct influence on growth from linear perturbations. By applying a range of values for $\nu$ and $\eta$ to an initial state consisting of fully developed turbulence in the presence of a background toroidal field, we investigate their effects in the fully nonlinear system. Here, increased viscosity enhances the turbulence, and the turbulence decays only if the resistivity is above a critical value; turbulence can be sustained even when $P_{\mathrm{m}}<1$, in contrast to the zero net field model. While we find preliminary evidence that the stress converges to a small range of values when $\nu$ and $\eta$ become small enough, the influence of dissipation terms on MRI-driven turbulence for relatively large $\eta$ and $\nu$ is significant, independent of field geometry.
\end{abstract}

Subject headings: accretion, accretion disks - black hole physics - (magnetohydrodynamics:) MHD 


\section{Introduction}

Disk accretion is a fundamental process of many astrophysical phenomena, from nearby young stellar objects to immensely luminous distant quasars. Understanding the mechanism for removing angular momentum from a fluid element, thereby allowing accretion to occur, is essential to understanding these systems. Orbiting, magnetized gas is unstable to the magnetorotational instability (MRI) (Balbus \& Hawley 1991, 1998); all that is required is a subthermal magnetic field sufficiently coupled to differentially rotating gas with a negative outward angular velocity gradient. The MRI leads to turbulent flow, resulting in Maxwell and Reynolds stresses that efficiently transport angular momentum and drive accretion. However, it is still uncertain what determines the amplitude of the magnetic energy and stress in saturated MRI-driven turbulence.

Because linear analysis can offer only limited guidance, numerical simulations have been used to investigate the properties of MRI-driven turbulence. Most simulations of the MRI employ the shearing box approximation, in which the simulation domain consists of a local corotating patch of accretion disk, small enough to expand the MHD equations into Cartesian coordinates and ignore curvature terms (see Hawley et al. 1995). This approximation, in its simplest form, reduces the problem to its basic ingredients: differential rotation and magnetized fluid. It is hoped that a more complete understanding of this simple model will provide insights into the mechanisms that determine the stress levels in astrophysical systems.

The first shearing box simulations (Hawley et al. 1995, 1996) found that the presence of a net magnetic field and its orientation play a role in setting the amplitude of the MRI turbulence. A net magnetic field results from currents located outside of the computational domain and cannot change as a result of the evolution. For zero net field simulations, on the other hand, complete decay of the field is possible. Net vertical fields gave the largest turbulent energies, with the energy level approximately proportional to the background field. Net toroidal fields behave similarly, but with a smaller energy for the same background field strength. Zero net field simulations saturated at levels comparable to those seen in net toroidal field cases.

In subsequent years, there have been many shearing box simulations which confirm these qualitative behaviors, but the factors that determine the amplitude of the turbulent energy still remain uncertain. Some studies have examined numerical effects such as computational domain size and resolution, and others have looked at physical parameters such as background field strength and gas pressure. An investigation of the influence of gas pressure carried out by Sano et al. (2004), for example, found an extremely weak pressure dependence. Even here, the influence of the gas pressure depends on the magnetic field geometry. 
Blackman et al. (2008) examined the results of an ensemble of shearing box simulations taken from the literature and found that $\alpha \beta$ is generally constant, where $\alpha$ is the total stress divided by the gas pressure, and $\beta$ is the ratio of thermal to magnetic pressure. In other words, stress is proportional to magnetic energy.

A physical influence that has, until recently, received less attention is physical dissipation, namely shear viscosity $\nu$ and Ohmic resistivity $\eta$. The linear dispersion relation for the vertical field MRI in the presence of $\nu$ and $\eta$ was derived by Balbus \& Hawley (1998). Both terms can reduce the effectiveness of the MRI. In the linear regime, viscosity damps the MRI growth rates and changes the wavelength of the fastest growing mode, but does not alter the wavenumbers that are unstable. Resistivity introduces a cutoff on the unstable wavelengths where the resistive diffusion time becomes comparable to the MRI growth time (see, e.g., the discussion in Masada \& Sano 2008). Nonaxisymmetric MRI modes with Ohmic resistivity were examined by Papaloizou \& Terquem (1997). They found that resistivity reduces the amplification of such modes, and if large enough, can stabilize the toroidal field MRI.

Simulations by Hawlev et al. (1996), Sano et al. (1998), Fleming et al. (2000), Sano \& Inutsuka (2001), Ziegler \& Rüdiger (2001), and Sano \& Stone (2002) have investigated the influence of a nonzero Ohmic resistivity on the saturated state. The main result of these studies is that increasing the resistivity leads to a decrease in turbulence, independent of the magnetic field configuration. In zero net field models, the effect of resistivity on the turbulence is larger than one might expect from the linear MRI relation (Fleming et al. 2000), with the turbulence decaying to zero for relatively low values of resistivity.

Recently, the work of Fromang et al. (2007) (hereafter F07) and Lesur \& Longaretti (2007) has sparked new interest in the effects of non-ideal MHD on the MRI. F07 showed that both resistivity and viscosity are important in determining the stress level in MRI turbulent flows with zero net magnetic field. Lesur \& Longaretti (2007) came to the same conclusion for models with a net vertical field. The results were characterized in terms of the magnetic Prandtl number, defined as $P_{\mathrm{m}}=\nu / \eta$. In these simulations, the saturation level increases with increasing $P_{\mathrm{m}}$. F07 also find that for the zero net field case, there exists a $P_{\mathrm{m}}$ below which the turbulence dies out, and that this critical $P_{\mathrm{m}}$ decreases with decreasing viscosity (at least for the range in viscosity and resistivity examined in the paper).

One magnetic field geometry that has not yet been explored with both physical resistivity and viscosity is that of a net toroidal field. Such fields could be the most relevant to astrophysical disks. Following the arguments of Guan et al. (2009) and references therein, both global and local disk simulations as well as observations of disk galaxies show a dominance of toroidal field over other field components. Indeed, the background shear flow naturally creates toroidal field from radial field. It seems likely that any given region of an 
accretion disk will contain some net azimuthal field.

In this paper, we perform the first investigation of the toroidal field MRI in the presence of both viscosity and resistivity and compare the outcomes with those obtained for zero net and net vertical field simulations. The structure of the paper is as follows. In $\S 2$, we describe our algorithm, parameters, and tests of our viscosity and resistivity implementation. For comparison purposes, we reexamine the simulations of F07 with our code in $\S$, Our main results, focusing on the toroidal field simulations, are presented in $\S$ 4. We wrap up with our discussion and conclusions in $\S 5$.

\section{Numerical Simulations}

In this study, we use the Athena code, a second-order accurate Godunov scheme for solving the equations of MHD in conservative form using the dimensionally unsplit corner transport upwind (CTU) method of Colella (1990) coupled with the third-order in space piecewise parabolic method (PPM) of Colella \& Woodward (1984) and a constrained transport (CT; Evans \& Hawley 1988) algorithm for preserving the $\nabla \cdot \boldsymbol{B}=0$ constraint. A description of this algorithm and various test problems is given in Gardiner \& Stone (2005b), Gardiner \& Stone (2008), and Stone et al. (2008). For the present study, we have added physical dissipation in the form of a constant kinematic shear viscosity and Ohmic resistivity using operator splitting, as described in more detail below. Bulk viscosity is ignored.

The shearing box approximation is a model for a local region of a disk orbiting at a radius $R$ whose size is small compared to this radius, allowing us to expand the equations of motion in Cartesian form, as described in detail by Hawley et al. (1995). The box corotates with an angular velocity $\Omega$ corresponding to the value at the center of the box. The shearing box evolution equations with viscosity and resistivity are given by Balbus \& Hawlev (1998) and are:

$$
\begin{gathered}
\frac{\partial \rho}{\partial t}+\nabla \cdot(\rho \boldsymbol{v})=0, \\
\frac{\partial \rho \boldsymbol{v}}{\partial t}+\nabla \cdot(\rho \boldsymbol{v} \boldsymbol{v}-\boldsymbol{B B})+\nabla\left(P+\frac{1}{2} B^{2}\right)=2 q \rho \Omega^{2} \boldsymbol{x}-2 \boldsymbol{\Omega} \times \rho \boldsymbol{v}+\nabla \cdot(\rho \nu \nabla \boldsymbol{v})+\nabla\left(\frac{1}{3} \rho \nu \nabla \cdot \boldsymbol{v}\right), \\
\frac{\partial \boldsymbol{B}}{\partial t}=\nabla \times(\boldsymbol{v} \times \boldsymbol{B}-\eta \nabla \times \boldsymbol{B}),
\end{gathered}
$$

where $\rho$ is the mass density, $\rho \boldsymbol{v}$ is the momentum density, $\boldsymbol{B}$ is the magnetic field, $P$ is the gas pressure, and $q$ is the shear parameter, defined as $q=-d \ln \Omega / d \ln R$. We use $q=3 / 2$, appropriate for a Keplerian disk. We assume an isothermal equation of state $P=\rho c_{\mathrm{s}}^{2}$, where 
$c_{\mathrm{S}}$ is the isothermal sound speed. Shear viscosity and Ohmic resistivity are denoted by $\nu$ and $\eta$ respectively. Note that our system of units has the magnetic permeability $\mu=1$. The first source term on the right-hand side of equation (2) corresponds to tidal forces (gravity and centrifugal) in the corotating frame. The second source term in equation (2) is the Coriolis force. Finally, we have omitted the vertical component of gravity, making these "unstratified" shearing box simulations.

Adapting the Athena code to the shearing box problem requires adding the tidal and Coriolis force terms and implementing the shearing-periodic boundary conditions at the $x$ boundaries. The source terms are included in the algorithm in a directionally unsplit manner, consistent with the CTU algorithm. We do not use the Crank-Nicholson method of Gardiner \& Stone (2005a) that ensures precise conservation of epicyclic energy. We have found this added complexity to be unnecessary for simulations dominated by the MRI (see arguments in Simon et al. 2009). The shearing-periodic boundary conditions are described in Simon et al. (2009). Quantities are linearly reconstructed in the ghost zones from appropriate zones in the physical domain that have been shifted along $y$ to account for the shear across the boundaries. Furthermore, the $y$ momentum is adjusted to account for the shear across the $x$ boundaries as fluid moves out one boundary and enters at the other.

Note that to preserve a quantity to machine precision across a grid boundary such as the shearing-periodic boundary (or a boundary between different grids in a mesh-refinement scheme), it is necessary to reconstruct a quantity's flux (or for the magnetic field, the electromotive force, EMF) at the boundary, rather than the quantity itself (see Simon et al. 2009). To conserve total vertical field flux, for example, we reconstruct the $y$ EMF at the $x$ boundaries. This is essential, given the strong effect that a net vertical field has on the turbulence level. The perfect conservation of net toroidal flux is not as important, and as ensuring its precise conservation involves a more complex procedure, we allow the $B_{y}$ flux to be conserved only to truncation level. Note that in our simulations initialized with a net toroidal field, this truncation error results in a loss of net $B_{y}$ flux from the domain; 5-10\% of the initial toroidal field is lost per 100 orbits for our high resolution, sustained turbulence simulations. This corresponds to a background $\beta$ value of $\sim 110-120$ at 100 orbits. While this truncation error does not appear to have any significant affect on the turbulent energy levels in our simulations, it may become important to conserve $B_{y}$ to roundoff level for longer evolution times. The radial flux, $B_{x}$, will automatically be conserved to machine precision because its evolution is determined by EMFs on the periodic $y$ and $z$ boundaries. We also reconstruct the density flux on the $x$ boundaries to conserve the total mass in the domain to machine precision. The systematic difference between the calculation of outward and inward fluxes at the shearing $x$ boundaries can lead to a loss of mass from the grid (Simon et al. 2009). We do not reconstruct momentum fluxes at the boundaries as the source terms will 
prevent roundoff level conservation of momentum.

Both the viscosity and resistivity are added via operator splitting; the fluid variables updated from the CTU integrator are used to calculate the viscous and resistive terms on the right-hand side of equations (2) and (3). These terms are discretized in a flux-conservative manner consistent with the Athena algorithm. In particular, the third and fourth terms on the right-hand side of equation (2) are written so that $\rho \nu \nabla \boldsymbol{v}$ and $(1 / 3) \rho \nu \nabla \cdot \boldsymbol{v}$ are defined as fluxes at the cell faces. Taking the divergence of the third term and the gradient of the fourth term via finite-differencing ensures that momentum conservation is not violated by the viscous terms. The resistive contribution to the induction equation is added in a manner consistent with the EMFs; the term $\eta \nabla \times \boldsymbol{B}$ is computed at cell corners to ensure that when differenced via the curl operator, $\nabla \cdot \boldsymbol{B}=0$ is maintained. Note that this resistive contribution to the EMF must also be reconstructed at the shearing-periodic boundaries in order to preserve $B_{z}$ precisely.

More generally, the viscous term in equation (2) can be written in a flux-conservative manner as $\nabla \cdot \boldsymbol{T}$ where $\boldsymbol{T}$ is a viscous stress tensor defined as

$$
T_{i j}=\rho \nu\left(\frac{\partial v_{i}}{\partial x_{j}}+\frac{\partial v_{j}}{\partial x_{i}}-\frac{2}{3} \delta_{i j} \nabla \cdot \boldsymbol{v}\right),
$$

where the indices refer to the spatial components (Landau \& Lifshitz 1959). For simplicity, we have used the form as in equation (2) which is equivalent to equation (4) assuming that $\rho \nu$ is spatially constant. We have performed a few shearing box experiments with both implementations, and find no significant differences in turbulent stress evolution. In particular, we restarted a few simulations using the form in equation (4). We found that the volume-averaged magnetic energies are initially indistinguishable between the two approaches. Due to the chaotic nature of the MRI, the two curves eventually diverge, but nevertheless maintain the same time average.

The addition of viscosity and resistivity places an additional constraint on the time step,

$$
\Delta t=C_{o} \operatorname{MIN}\left(\Delta t_{\mathrm{CTU}}, 0.75 \frac{\Delta^{2}}{8 / 3 \nu}, 0.75 \frac{\Delta^{2}}{2 \eta}\right)
$$

where $C_{o}$ is the CFL number $\left(C_{o}=0.4\right.$ here $), \Delta t_{\mathrm{CTU}}$ is the time step limit from the main integration algorithm (see Stone et al. 2008), and $\Delta$ is the minimum grid spacing, $\Delta=$ $\operatorname{MIN}(\Delta x, \Delta y, \Delta z)$. Several three-dimensional tests of viscosity and resistivity revealed that if the viscous or resistive time step is close to $\Delta t_{\mathrm{CTU}}$, the evolution becomes numerically unstable. This problem was remedied by multiplying the viscous and resistive time steps by 0.75 . The additional $4 / 3$ factor in the denominator of the viscous time step results from 
the last term on the right-hand side of equation (2). This can be most easily understood by considering a one-dimensional problem, in which case the effective $\nu$ value increases by a factor of $4 / 3$ due to the compressibility term. Therefore, the effective $\nu$ that goes into the time step calculation is taken as $(4 / 3) \nu$. Note that most of our simulations will have $\nu$ and $\eta$ sufficiently small that the viscous and resistive time steps are large compared to $\Delta t_{\mathrm{CTU}}$. In fact, only the simulations with the largest values of $\eta$ and $\nu$ reach the diffusion limit on $\Delta t$.

\subsection{Tests of Physical Dissipation}

We performed a number of problems to test the implementation of viscosity and resistivity within Athena. Resistivity was tested by solving the diffusion of a current sheet along one dimension; a uniform magnetic field is initialized with a change in sign across one grid zone. This problem has a simple analytic solution (see e.g., Komissarov 2007). The agreement between the numerical and analytic solution was excellent. By replacing the magnetic field with a uniform velocity flow, the identical test can be performed for the viscosity. Again, the numerical solution agreed with the analytic solution.

Next, we initialized a uniform vertical magnetic field in a shearing box with nonzero viscosity and resistivity and measured the growth of various MRI modes in the linear regime. We compared the measured values with those from analytic linear theory (see e.g., Masada \& Sano 2008; Pessah \& Chan 2008) and found excellent agreement for a wide range in viscosity and resistivity.

Finally, we examined the propagation of small amplitude, isothermal sound and Alfvén waves in the presence of viscosity and resistivity. Again, the numerical solution can be compared directly to an analytic solution. These tests were done in one, two, and three dimensions; in the multidimensional tests, the propagation direction of the wave was chosen to be along the grid diagonal. The resistivity was tested via the decay of the Alfvén waves, and the viscosity was tested via the decay of the sound waves. The error as a function of $x$ resolution for two of these tests is given in Fig. 1, The error is calculated from the square root of the sum of the squared errors in the density and momenta (for the sound wave) and the density, momenta, and magnetic field (for the Alfvén wave). The solution to each wave converges at a rate very close to second order, shown by the dashed line. 


\subsection{Shearing Box Parameters}

The shearing box used in this study has radial size $L_{x}=1$, azimuthal size $L_{y}=4$, and vertical size $L_{z}=1$. Most of the simulations presented here use $128 \times 200 \times 128$ equally spaced grid zones; some simulations use half the number of zones in each direction. The initial velocity is $\boldsymbol{v}=-q \Omega x \hat{\boldsymbol{y}}$, with $q=3 / 2, \Omega=0.001$, and $-L_{x} / 2 \leq x \leq L_{x} / 2$. The isothermal sound speed is $c_{\mathrm{s}}=\Omega H$ where $H$ is the scale height. With $L_{z}=H$, we have $c_{\mathrm{s}}=L_{z} \Omega$, and with $\rho=1$, the initial pressure is $P=\rho \Omega^{2} L_{z}^{2}=10^{-6}$.

The dissipation terms $\nu$ and $\eta$ are parameterized in terms of the Reynolds number,

$$
R e \equiv \frac{c_{\mathrm{s}} H}{\nu},
$$

the magnetic Reynolds number,

$$
R m \equiv \frac{c_{\mathrm{s}} H}{\eta},
$$

and the magnetic Prandtl number,

$$
P_{\mathrm{m}} \equiv \frac{\nu}{\eta}=\frac{R m}{R e} .
$$

Since the properties of the MRI are more directly determined by the Alfvén speed rather than the sound speed, another useful dimensionless quantity is the Elsasser number,

$$
\Lambda \equiv \frac{v_{\mathrm{A}}^{2}}{\eta \Omega},
$$

where $v_{\mathrm{A}}$ is the Alfvén speed. With $c_{\mathrm{s}}=\Omega H$ and $\beta=2 c_{\mathrm{s}}^{2} / v_{\mathrm{A}}^{2}$, we can relate $R m$ to $\Lambda$,

$$
\Lambda=\frac{2}{\beta} R m .
$$

In addition to the explicit dissipation terms, there will also be some effective diffusion due to numerical effects. Generally speaking, numerical diffusion will not behave in the same manner as physical diffusion (e.g., it is not a simple function of a gradient in field or velocity); numerical diffusion generally has a much stronger effect at small scales than at large scales. Also the effects of numerical diffusion may be different from one type of simulation to 
another. By calculating numerical losses at high wavenmbers in Fourier space and modeling those as if they were physical viscosity and resistivity, Simon et al. (2009) quantified the numerical dissipation of Athena. They found that the effective $R m$ for the zero net field and net $\mathrm{z}$ field simulations at $N_{x}=128$ were 20000 and 8000 respectively, and 7000 and 5000 for $N_{x}=64$. The effective $P_{\mathrm{m}}$ is $\sim 2$ for these simulations. Since numerical dissipation is problem-dependent, these numbers should be regarded as estimates, and their values will likely be somewhat different in different applications. However, they serve as a guideline for including physical dissipation. In the present study, numerical and physical dissipation may be comparable at large wavenumbers for $R e, R m \gtrsim 10000$. The physical dissipation in some of our simulations may fall into this marginally resolved regime. Nevertheless, we can explore a large enough range in $R e$ and $R m$ values to observe clear effects due to viscosity and resistivity.

\section{Zero Net Flux Simulations}

Fromang \& Papaloizou (2007) and Pessah et al. (2007) presented the surprising result that for zero net field shearing box simulations without any explicit dissipation terms, the steady-state turbulent energy decreases with increasing grid resolution. Simon et al. (2009) obtained the same result for zero net field simulations without explicit dissipation using the Athena code. These results pointed to the importance of including explicit dissipation terms in such simulations.

F07 showed that turbulent activity is strongly influenced by these dissipation terms; the saturated stress increases with increasing $P_{\mathrm{m}}$. Here we return to the zero net field problem and include the dissipative terms to compare with the results of F07. The simulations are initialized with $\boldsymbol{B}=\sqrt{2 P / \beta} \sin \left[\left(2 \pi / L_{x}\right) x\right] \hat{\boldsymbol{z}}$ where $\beta=400$. These runs are labeled SZ for sinusoidal z-field and have resolution $N_{x}=128, N_{y}=200, N_{z}=128$. The viscosity and resistivity in these simulations are chosen to reproduce the calculations of F07. The initial state is perturbed in each grid zone with random fluctuations in $\rho$ at amplitude $\delta \rho / \rho=0.01$. The SZ simulations are listed in Table 1, The column labeled "Turbulence?" states whether or not the turbulence was sustained in a given simulation. The column labeled " $\alpha$ " gives the resulting turbulent stress in terms of the dimensionless value $\alpha \equiv\left\langle\left\langle\rho v_{x} \delta v_{y}-B_{x} B_{y}\right\rangle\right\rangle / P_{o}$, with $\delta v_{y} \equiv v_{y}+q \Omega x . P_{o}$ is the initial gas pressure and the double bracket denotes a time and volume average. The time average is calculated from orbit 20 until the end of the simulation, and as is the case throughout this paper, volume average refers to an average over the entire simulation domain.

The results of these simulations are consistent with those of F07. For example, F07 lists 
$\alpha$ values for a $R e=3125$ and $R m=12500$ model run with four different codes, including ZEUS. These values range from $\alpha=0.0091$ to 0.011 ; we obtain 0.013 . The increase in turbulent energy levels with $P_{\mathrm{m}}$ is demonstrated by a series of simulations with the same $R m$ and increasing viscosity. For example, for a constant $R m \approx 12800$ (some of the simulations had $R m=12800$ while others had $R m=12500$; see F07), $P_{\mathrm{m}}$ values were varied by factors of 2 from 1 to 16 . Sustained turbulence was seen for $P_{\mathrm{m}} \geq 4$ with $\alpha$ values increasing from 0.0091 for $P_{\mathrm{m}}=4$ to 0.019 and 0.044 for $P_{\mathrm{m}}=8$ and 16 respectively. The Athena runs have $\alpha$ values of $0.013,0.026$, and 0.046 . These data are plotted in Fig. 2 , which shows that the increase in $\alpha$ with $P_{\mathrm{m}}$ is nearly linear.

The largest differences between the Athena simulations and the ZEUS simulations of F07 lie in the marginally turbulent cases. For example, we find decaying turbulence for $R e=1600$, $P_{\mathrm{m}}=4$, whereas ZEUS produces sustained turbulence for these parameters. Figure 3 shows the volume-averaged magnetic energy density normalized by the gas pressure versus time for the three $P_{\mathrm{m}}$ values at $R e=1600$. The lowest $P_{\mathrm{m}}$ simulation decays quite rapidly, whereas the $P_{\mathrm{m}}=4$ case takes roughly 60 orbits to decay. Differences in the numerical properties of Athena and ZEUS might account for these results, given the sensitivity to numerical factors as shown by zero net field simulations. We also note that we use a slightly larger domain size in $y$ than in F07. The boundary in parameter space between sustained turbulence and decay is unlikely to be hard and fast, and detailed numerical surveys that attempt to define that boundary are probably not warranted. Some such studies may, however, provide additional insights into the sensitivity of the MRI turbulence to specific numerical factors.

Table 1. Zero Net Flux Simulations

\begin{tabular}{c|ccccc}
\hline \hline Label & $R e$ & $P_{\mathrm{m}}$ & $R m$ & Turbulence? & $\alpha$ \\
\hline SZRe800Pm4 & 800 & 4 & 3200 & No & - \\
SZRe800Pm8 & 800 & 8 & 6400 & Yes & 0.031 \\
SZRe800Pm16 & 800 & 16 & 12800 & Yes & 0.046 \\
SZRe1600Pm2 & 1600 & 2 & 3200 & No & - \\
SZRe1600Pm4 & 1600 & 4 & 6400 & No & - \\
SZRe1600Pm8 & 1600 & 8 & 12800 & Yes & 0.026 \\
SZRe3125Pm1 & 3125 & 1 & 3125 & No & - \\
SZRe3125Pm2 & 3125 & 2 & 6250 & No & - \\
SZRe3125Pm4 & 3125 & 4 & 12500 & Yes & 0.013 \\
\hline
\end{tabular}




\section{Toroidal Field Simulations}

To examine the effect of viscosity and resistivity on the MRI with a net toroidal field, we have run a series of simulations initialized with $\boldsymbol{B}=\sqrt{2 P / \beta} \hat{\boldsymbol{y}}$, where $\beta=100$, and with varied $R e$ and $R m$ values. $R e$ ranges from 100 to 25600, and $P_{\mathrm{m}}$ ranges from 0.25 to 16 (though, in some simulations, we set either $\eta$ or $\nu$ equal to zero). We will consider the influence of the physical dissipation terms on two types of problems: the linear MRI growth regime, and fully nonlinear turbulence.

\subsection{The Linear Regime}

The linear nonaxisymmetric MRI was first examined by Balbus \& Hawley (1992). For nonaxisymmetric modes, the MRI tends to be most robust in the presence of a poloidal field. However, even the purely toroidal field case is unstable, athough, as emphasized by Balbus \& Hawley (1992), that case is somewhat singular. As always with the ideal MRI, the most unstable mode has $\mathbf{k} \cdot \mathbf{v}_{\mathbf{A}} \simeq \Omega$. The linear analysis is complicated by the background shear which causes radial wavenumbers to evolve with time. Amplification of a given mode occurs when the wavenumber ratio $k / k_{z}$ goes through a minimum as the radial wavenumber swings from leading to trailing. In general, the purely toroidal MRI favors high $k_{z}$ wavenumbers and small values of $k_{y} / k_{z}$, in contrast to the vertical field MRI where the wavenumber $k_{z}$ of the most unstable mode is determined by the Alfvén speed.

Papaloizou \& Terquem (1997) examined the toroidal field MRI with the addition of resistivity. They point out that because $k_{x}$ grows arbitrarily large, all linear modes will eventually damp out in the presence of resistivity. For small enough resistivities, however, there can be a period of growth when $k_{x} \sim 0$. For the MRI to become self-sustaining, this growth has to continue long enough for the perturbations to reach nonlinear amplitudes. Resistivity is also particularly important for the pure toroidal field MRI because large $k_{z}$ is favored for mode growth. Equation (32) of Papaloizou \& Terquem (1997) provides an approximate condition for transient amplification of the MRI in the presence of resistivity. For Keplerian shear and for modes where $\mathbf{k} \cdot \mathbf{v}_{\mathbf{A}} \sim \Omega$, this reduces to the condition

$$
k_{z}^{2} \eta \sim \Omega
$$

In other words, there is no amplification of modes for which the diffusion time is comparable to the orbital frequency. Although viscosity was not included in the analysis, one might expect it to be similarly influential.

Simulations of the linear growth of the MRI in the presence of resistivity for a purely 
toroidal $\beta=100$ initial field were first carried out by Fleming et al. (2000) using a ZEUS code with an adiabatic equation of state. For this field strength, the critical MRI wavelength in the azimuthal direction is $2 \pi v_{A} / \Omega \approx H$. They found field decay for a $R m=2000$ simulation, but field growth to turbulent saturation for $R m=5000$ and above.

In this section, we follow the growth of the MRI in a shearing box with a purely toroidal field while including both resistivity and viscosity. The system is seeded within each grid zone with random perturbations in $\rho$ at amplitude $\delta \rho / \rho=0.01$. The simulations were run at two resolutions, $N_{x}=64, N_{y}=100, N_{z}=64$ and $N_{x}=128, N_{y}=200, N_{z}=128$ and are labelled YL for $y$-field, linear regime. In this standard set of simulations, the range of $R e$ examined runs from 800 to 25600, and the range of $R m$ is from 400 to 102400. Table 2 lists these simulations. The last two columns state whether or not MRI growth is observed for the $N_{x}=64$ and $N_{x}=128$ resolutions, respectively. A dash in either of these columns means that the simulation was not run at that particular resolution. MRI growth is defined by the evolution of the volume-averaged magnetic and kinetic energy components. A simulation is considered to have zero growth if after 20-40 orbits, the various energy components are either decaying or constant in time without any indication of exponential increase. Growth to saturation is observed in cases when $R e$ and $R m$ are at 6400 and above.

Clearly, a sufficiently large viscosity or resistivity can inhibit growth. But what about the very high or very low $P_{\mathrm{m}}$ limits? To approach that question, we carried out simulations where only $\nu$ or $\eta$ was nonzero. These experiments were done at the $N_{x}=64$ resolution. In our first experiments, we set $\eta$ to zero and $R e$ to 100 and 800 . The $R e=800$ run showed growth to saturation, but the $R e=100$ case had no growth. Next we set $\nu$ to zero and $R m$ to 800 and 1600 . The lower resistivity $(R m=1600)$ grew to saturation, whereas the higher resistivity $(R m=800)$ did not. Although the existence of a critical $R m$ value is consistent with the results of Fleming et al. (2000), the value of $R m$ at which growth is prevented is smaller here than what they found. We note that there remains unavoidable numerical dissipation associated with grid scale effects, which will make the value of a critical $R m$ obtained through simulations somewhat dependent on algorithm and resolution.

The effect of numerical resolution is not necessarily obvious. Consider model YLRe3200Pm2, which has $R e=3200$ and $R m=6400$, and model YLRe6400Pm0.5, which has these values reversed. In both cases, the $N_{x}=64$ simulations show growth but the $N_{x}=128$ models do not. One difference between the two resolutions is in the initial perturbations. While the density perturbations have the same amplitude in both resolutions, the higher resolution initial density is given power at smaller scales because the perturbations are applied to each grid zone. This leads to a smaller amplitude for each Fourier mode. Does this account for the difference seen in these two resolutions? To investigate this, we ran both $N_{x}=64$ versions of 
YLRe3200Pm2 and YLRe6400Pm0.5 with initial perturbations of amplitude $\delta \rho / \rho=0.005$ and $\delta \rho / \rho=0.001$. Note that these amplitudes lead to comparable $(\delta \rho / \rho=0.005)$ or smaller $(\delta \rho / \rho=0.001)$ amplitude modes in Fourier space compared to the $\delta \rho / \rho=0.01$ initialized modes at the higher resolution. Neither of the smaller amplitude YLRe3200Pm2 simulations showed any growth (as of 20-30 orbits), but both YLRe6400Pm0.5 simulations showed growth to saturation.

From these experiments it seems that the effects of viscosity and resistivity are comparable and that the transition region between decay and growth to turbulence lies between Reynolds numbers of 3200 and 6400 for $P_{\mathrm{m}}$ near unity. This corresponds to a critical vertical wavelength, defined in terms of equation (11), of $\lambda_{c} / H \sim 2 \pi / R m^{1 / 2}=0.111$ and 0.079 , respectively. As viscosity (resistivity) is increased, MRI growth can be achieved by decreasing the resistivity (viscosity). This trend only works up to certain limits; if either the viscosity

or resistivity is large enough, MRI growth is completely quenched, independent of the value of the other dissipation term.

\subsection{The Nonlinear Regime}

Of potentially greater interest than the linear MRI regime is the effect of viscosity and resistivity on fully developed MRI-driven turbulence. To study this nonlinear regime, we begin with model YLRe25600Pm4, a simulation with $R e=25600$ and $P_{\mathrm{m}}=4$ at $N_{x}=128$, $N_{y}=200, N_{z}=128$ (Table 21) that was run to 59 orbits in time. The MRI grows and the flow becomes fully turbulent. Averaging from $t=15$ to 59 orbits gives a stress value of $\alpha=0.05$. We use this simulation at $t=36$ orbits to initialize a series of simulations with different values of $R e$ and $R m$. These runs are labelled YN for y-field, nonlinear regime, and they are all run to 200 orbits, except for simulation YNRe12800Pm0.25, which was run to 100 orbits. All the YN simulations are listed in Table 3 .

When evolving onward from orbit 36 with modified dissipation terms, a simulation shows a rapid readjustment followed by either sustained turbulence at a new amplitude or decay to smooth flow, depending on the new values of $R e$ and $R m$. The column labeled "Turbulence?" in Table 3 states whether or not the given simulation has sustained turbulence. Note that for $R m \gtrsim 1600$, the turbulence is sustained except for the relatively viscous $R e=400$ model. This critical $R m$ value is below the critical value obtained above for sustained growth in the linear regime when the resistivity and viscosity are comparable but near the critical $R m$ value in the linear regime in the absence of explicit viscosity. For simulations where turbulence is sustained, the column labeled " $\alpha$ " gives the time- and volume-averaged dimensionless stress, where the time average is calculated onward from orbit 50. 
Table 2. Toroidal Field Simulations Initialized from Linear Perturbations

\begin{tabular}{l|cccccc}
\hline \hline \multicolumn{1}{c|}{ Label } & $R e$ & $P_{\mathrm{m}}$ & $R m$ & $\Lambda$ & $N_{x}=64$ & $N_{x}=128$ \\
\hline YLRe800Pm0.5 & 800 & 0.5 & 400 & 8 & No & - \\
YLRe800Pm1 & 800 & 1 & 800 & 16 & No & - \\
YLRe800Pm2 & 800 & 2 & 1600 & 32 & No & - \\
YLRe800Pm4 & 800 & 4 & 3200 & 64 & No & - \\
YLRe800Pm8 & 800 & 8 & 6400 & 128 & No & - \\
YLRe1600Pm0.5 & 1600 & 0.5 & 800 & 16 & No & - \\
YLRe1600Pm1 & 1600 & 1 & 1600 & 32 & No & - \\
YLRe1600Pm2 & 1600 & 2 & 3200 & 64 & No & - \\
YLRe1600Pm4 & 1600 & 4 & 6400 & 128 & No & - \\
YLRe1600Pm8 & 1600 & 8 & 12800 & 256 & No & - \\
YLRe3200Pm0.5 & 3200 & 0.5 & 1600 & 32 & No & No \\
YLRe3200Pm1 & 3200 & 1 & 3200 & 64 & No & No \\
YLRe3200Pm2 & 3200 & 2 & 6400 & 128 & Yes & No \\
YLRe3200Pm4 & 3200 & 4 & 12800 & 256 & - & Yes \\
YLRe6400Pm0.5 & 6400 & 0.5 & 3200 & 64 & Yes & No \\
YLRe6400Pm1 & 6400 & 1 & 6400 & 128 & Yes & Yes \\
YLRe6400Pm2 & 6400 & 2 & 12800 & 256 & Yes & Yes \\
YLRe6400Pm4 & 6400 & 4 & 25600 & 512 & Yes & Yes \\
YLRe12800Pm0.5 & 12800 & 0.5 & 6400 & 128 & Yes & Yes \\
YLRe12800Pm1 & 12800 & 1 & 12800 & 256 & Yes & Yes \\
YLRe12800Pm2 & 12800 & 2 & 25600 & 512 & Yes & Yes \\
YLRe12800Pm4 & 12800 & 4 & 51200 & 1024 & Yes & Yes \\
YLRe25600Pm0.5 & 25600 & 0.5 & 12800 & 256 & Yes & Yes \\
YLRe25600Pm1 & 25600 & 1 & 25600 & 512 & Yes & Yes \\
YLRe25600Pm2 & 25600 & 2 & 51200 & 1024 & Yes & Yes \\
YLRe25600Pm4 & 25600 & 4 & 102400 & 2048 & Yes & Yes \\
\hline
\end{tabular}


The column labeled " $\langle\langle\Lambda\rangle\rangle$ " gives a time- and volume-averaged $\Lambda$ value in the final state of each simulation. Unlike $R m, \Lambda$ will change with the evolving magnetic field strength. Beginning with equation (10), we write

$$
\beta=\frac{2 c_{\mathrm{s}}^{2}\langle\rho\rangle}{\left\langle B^{2}\right\rangle}
$$

to give

$$
\langle\Lambda\rangle=\frac{R m}{c_{\mathrm{s}}^{2}} \frac{\left\langle B^{2}\right\rangle}{\langle\rho\rangle},
$$

where the angled brackets denote a volume average. One could also volume-average the square of the Alfvén speed in the calculation of $\beta$ instead of averaging $B^{2}$ and $\rho$ separately (e.g., $\beta=2 c_{\mathrm{s}}^{2} /\left\langle v_{\mathrm{A}}^{2}\right\rangle$ ). We have calculated $\langle\Lambda\rangle$ using both types of averages for several frames in the saturated state of a few simulations. We have found at most a factor of 2 difference between the different calculations. Since $\left\langle B^{2}\right\rangle$ varies by a similar factor in the saturated state (see Fig. (4), this factor of 2 difference is within the uncertainty of $\Lambda$ at any given time. The time-average of the volume-averaged Elsasser number, $\langle\langle\Lambda\rangle\rangle$, as given in the table, is calculated from orbit 50 until the end of the simulation. For the decayed turbulence simulations in which the turbulence has not fully decayed by orbit 50 , the time average is calculated onward from a point at which the volume-averaged magnetic energy is constant in time. Note that for these decayed turbulence simulations, $\langle\langle\Lambda\rangle\rangle$ should equal the $\beta=100$ value associated with the net toroidal field, as given in Table 2. However, because of the evolution of the net $B_{y}$ (see $\S 2$ ), the value of $\langle\langle\Lambda\rangle\rangle$ after the turbulence has decayed will be slightly different than the $\beta=100$ value.

Since the magnetic field varies within the domain, the local value of $\Lambda$ can also vary from the overall average. Histograms showing the number of grid zones with $v_{\mathrm{A}}^{2}$ of a certain value reveal that the percentage of grid zones that have $\Lambda<1$ is at most $\sim 0.01 \%$. For the sustained turbulence models, $\langle\langle\Lambda\rangle\rangle$ is typically on the order of $100-1000$; the smallest value for a run with sustained turbulence is 106, and the largest value associated with a run that decays is 30 .

The behavior of the MRI is often characterized by the vertical component of the Alfvén speed, and as such, we have also calculated the Elsasser number using only the vertical component of the magnetic field,

$$
\left\langle\Lambda_{z}\right\rangle=\frac{R m}{c_{\mathrm{s}}^{2}} \frac{\left\langle B_{z}^{2}\right\rangle}{\langle\rho\rangle},
$$


Table 3. Toroidal Field Simulations Initialized from Nonlinear Turbulence

\begin{tabular}{l|ccccccc}
\hline \hline \multicolumn{1}{c|}{ Label } & $R e$ & $P_{\mathrm{m}}$ & $R m$ & Turbulence? & $\alpha$ & $\langle\langle\Lambda\rangle\rangle$ & $\left\langle\left\langle\Lambda_{z}\right\rangle\right\rangle$ \\
\hline YNRe400Pm0.5 & 400 & 0.5 & 200 & No & - & 4 & - \\
YNRe400Pm1 & 400 & 1 & 400 & No & - & 8 & - \\
YNRe400Pm2 & 400 & 2 & 800 & No & - & 15 & - \\
YNRe400Pm4 & 400 & 4 & 1600 & No & - & 30 & - \\
YNRe400Pm8 & 400 & 8 & 3200 & Yes & 0.043 & 614 & 16.8 \\
YNRe400Pm16 & 400 & 16 & 6400 & Yes & 0.068 & 1983 & 58.2 \\
YNRe800Pm0.25 & 800 & 0.25 & 200 & No & - & 4 & - \\
YNRe800Pm0.5 & 800 & 0.5 & 400 & No & - & 8 & - \\
YNRe800Pm1 & 800 & 1 & 800 & No & - & 15 & - \\
YNRe800Pm2 & 800 & 2 & 1600 & Yes & 0.019 & 137 & 3.87 \\
YNRe800Pm4 & 800 & 4 & 3200 & Yes & 0.038 & 495 & 18.0 \\
YNRe800Pm8 & 800 & 8 & 6400 & Yes & 0.054 & 1413 & 56.2 \\
YNRe1600Pm0.5 & 1600 & 0.5 & 800 & No & - & 15 & - \\
YNRe1600Pm1 & 1600 & 1 & 1600 & Yes & 0.018 & 120 & 4.45 \\
YNRe1600Pm2 & 1600 & 2 & 3200 & Yes & 0.033 & 403 & 18.6 \\
YNRe1600Pm4 & 1600 & 4 & 6400 & Yes & 0.044 & 1120 & 52.6 \\
YNRe3200Pm0.5 & 3200 & 0.5 & 1600 & Yes & 0.016 & 106 & 4.53 \\
YNRe3200Pm1 & 3200 & 1 & 3200 & Yes & 0.025 & 314 & 16.4 \\
YNRe3200Pm2 & 3200 & 2 & 6400 & Yes & 0.035 & 860 & 47.4 \\
YNRe3200Pm4 & 3200 & 4 & 12800 & Yes & 0.043 & 2170 & 127 \\
YNRe6400Pm0.5 & 6400 & 0.5 & 3200 & Yes & 0.021 & 263 & 14.9 \\
YNRe6400Pm1 & 6400 & 1 & 6400 & Yes & 0.031 & 748 & 45.2 \\
YNRe6400Pm2 & 6400 & 2 & 12800 & Yes & 0.038 & 1880 & 118 \\
YNRe12800Pm0.25 & 12800 & 0.25 & 3200 & Yes & 0.021 & 262 & 15.8 \\
\hline
\end{tabular}


where the angled brackets denote a volume average. We have calculated the time average of this number, $\left\langle\left\langle\Lambda_{z}\right\rangle\right\rangle$, onward from orbit 50 for all the sustained turbulence YN simulations. This number is displayed in the last column of Table 3. The decayed turbulence simulations have $B_{z}$ approaching zero, and we do not calculate a vertical Elsasser number for these. Again, we calculated the vertical Elsasser number both by averaging $B_{z}^{2}$ and $\rho$ separately as well as by averaging the ratio $B_{z}^{2} / \rho$. We compared the two calculations for several frames and found at most a factor of 1.3 difference between them.

The $\left\langle\left\langle\Lambda_{z}\right\rangle\right\rangle$ values for the runs that have $R m$ closest to the critical value are on the order unity, with the smallest value being 3.87. As touched upon by Fleming et al. (2000), growth of the vertical field MRI is largely suppressed for $v_{\mathrm{A} z}^{2} /(\eta \Omega) \lesssim 1$ (i.e., for vertical Elsasser numbers less than unity). That we find $\left\langle\left\langle\Lambda_{z}\right\rangle\right\rangle \sim 1$ close to the "decayed turbulence" regime may suggest that the vertical field MRI plays an important role in the sustained nonlinear turbulence of these toroidal field simulations. One trend to note from these data is that the ratio of $\left\langle\left\langle\Lambda_{z}\right\rangle\right\rangle$ to $\langle\langle\Lambda\rangle\rangle$ increases with both decreasing $\nu$ and decreasing $\eta$; the vertical magnetic energy becomes a larger fraction of the total magnetic energy as either dissipation term is reduced.

The evolution of the magnetic energy in a typical set of simulations is shown in Fig. 4. For these runs, $R e=1600$ and $R m$ varies by factors of two from $R m=800$ to 6400 . The black line shows the initial evolution of YLRe25600Pm4, whose state at 36 orbits serves as the initial condition. It is clear that decreasing the resistivity (increasing the $P_{\mathrm{m}}$ number) enhances the saturation level, and for a large enough resistivity, the turbulence decays.

To quantify the dependence of the saturation amplitude on the dissipation coefficients, we plot the $\alpha$ values for the ensemble of simulations as a function of $R e, R m$ and $P_{\mathrm{m}}$. Figure 5 shows $\alpha$ versus $R m$; the color indicates $R e$ value, and the symbols correspond to the $P_{\mathrm{m}}$ value. The simulations with $\alpha=0$ are those where the turbulence decayed away, which include all simulations with $R m \leq 800$ and the $R e=400, R m=1600$ simulation. Overall there is a general trend of increasing $\alpha$ value with decreasing resistivity.

The dependence of $\alpha$ on $R e$ is shown in Fig. 6. Here the color indicates the $R m$ value, whereas $P_{\mathrm{m}}$ is again represented by a symbol. Evidently, if the resistivity is low enough, increasing the viscosity will increase the $\alpha$ values. However, consider the YN simulations with $R m=1600$. These simulations suggest that if the resistivity is close to some critical value, increasing the viscosity will cause the turbulence to decay. Another feature of note is that as $R e$ increases, the range of $\alpha$ for different $R m$ values becomes smaller, and $\alpha$ appears to converge to $\sim 0.02-0.04$ for all $R m$. This could indicate that as $\nu$ and $\eta$ decrease, their influence on the turbulence level might decrease. However, for large values of $R e$ or $R m$, the dissipation lengthscales are under-resolved, and higher resolution is needed to test this 
possibility.

We plot the dependence of $\alpha$ on $P_{\mathrm{m}}$ in Fig. 17. In this figure, the colors represent varying $R m$ while the symbols denote different $R e$ values. The clearest trend is that if $R m$ is large enough to sustain turbulence, increasing $P_{\mathrm{m}}$ leads to larger $\alpha$ values. Note that turbulence can be sustained even for $P_{\mathrm{m}}$ less than unity, if $R m$ is large enough. At constant $R m$, we find that $\alpha \propto R e^{\delta_{1}}$ with $\delta_{1}$ ranging from -0.1 to -0.3 (calculated by a linear fit to the data in log space for non-decayed turbulence simulations only). At constant $R e$ value, we find $\alpha \propto R m^{\delta_{2}}$ with $\delta_{2}$ in the range 0.4-0.8 and $\delta_{2}$ generally decreasing with increasing $R e$.

These results naturally lead to the question of why increasing $\nu$ or decreasing $\eta$ causes an increase in turbulence. Magnetic reconnection and dissipation of field lines, either due to an explicit resistivity or to grid-scale effects, presumably play the primary role in limiting the amplitude of the MHD turbulence. Balbus \& Hawley (1998) hypothesized that increased viscosity would inhibit reconnection by preventing velocity motions that would bring field together on small scales. When $P_{\mathrm{m}}>1$, the viscous length is greater than the resistive one, and magnetic field dissipation becomes less efficient, leading to an increase in turbulent stress (e.g., Balbus \& Henri 2008). If this hypothesis is correct, there may also be a change in the dissipation of kinetic and magnetic energy into heat. To investigate this possibility, we carry out an analysis of viscous and resistive heating for several of the simulations.

Consider the volume-averaged kinetic and magnetic energy evolution equations, equations (15) and (16) in Simon et al. (2009),

$$
\begin{aligned}
\dot{K}= & -\left\langle\nabla \cdot\left[\boldsymbol{v}\left(\frac{1}{2} \rho v^{2}+\frac{1}{2} B^{2}+P+\rho \Phi\right)-\boldsymbol{B}(\boldsymbol{v} \cdot \boldsymbol{B})\right]\right\rangle \\
& +\left\langle\left(P+\frac{1}{2} B^{2}\right) \nabla \cdot \boldsymbol{v}\right\rangle-\langle\boldsymbol{B} \cdot(\boldsymbol{B} \cdot \nabla \boldsymbol{v})\rangle-\dot{G}-Q_{\mathrm{k}},
\end{aligned}
$$

and

$$
\dot{M}=-\left\langle\nabla \cdot\left(\frac{1}{2} B^{2} \boldsymbol{v}\right)\right\rangle-\left\langle\frac{1}{2} B^{2} \nabla \cdot \boldsymbol{v}\right\rangle+\langle\boldsymbol{B} \cdot(\boldsymbol{B} \cdot \nabla \boldsymbol{v})\rangle-Q_{\mathrm{m}} .
$$

Here, $\dot{K}$ and $\dot{M}$ are the time derivatives of the volume-averaged kinetic and magnetic energies, respectively. The time derivative of the volume-averaged gravitational potential energy is given by $\dot{G}$, and $Q_{\mathrm{k}}$ and $Q_{\mathrm{m}}$ are the volume-averaged kinetic and magnetic energy dissipation rates, respectively. The gravitational potential is $\Phi=q \Omega^{2}\left(\frac{L_{x}^{2}}{12}-x^{2}\right)$. 
We determine $Q_{\mathrm{k}}$ and $Q_{\mathrm{m}}$ for select YN models by computing the time average of each of the source terms in the energy evolution equations using 200 data files equally spaced in time over 20 orbits. We assume $\dot{G}$ is zero in the time-average; the analysis of Simon et al. (2009) found $\dot{G}$ is always negligibly small. The time-derivatives, $\dot{K}$ and $\dot{M}$, are calculated by differentiating the volume-averaged kinetic and magnetic energy history data with respect to time and then sampling these data to the times associated with the data files. The dissipation terms $Q_{\mathrm{k}}$ and $Q_{\mathrm{m}}$, which include both physical and numerical effects, are the remainder after all the other terms are calculated.

Figure 8 shows the ratio of the time-average $\left\langle Q_{\mathrm{k}}\right\rangle$ to $\left\langle Q_{\mathrm{m}}\right\rangle$ as a function of $P_{\mathrm{m}}$ and $\alpha$ for select YN runs. The colors and symbols are the same as in Fig. 6. The time average is calculated from $t=70-90$ orbits for YNRe400Pm16 (black X) and YNRe12800Pm0.25 (blue circle), $t=110-130$ orbits for YNRe800Pm2 (green diamond) and YNRe800Pm8 (black square), and $t=110.6-130.6$ orbits for YNRe800Pm4 (blue triangle) and YNRe3200Pm4 (red triangle). The ratio of viscous to resistive heating generally increases as either $\alpha$ or $P_{\mathrm{m}}$ increases, although not monotonically. The relative heating ratio is not simply proportional to $P_{\mathrm{m}}$ as one might naively expect.

The data suggest a general relationship between saturated stress and $\left\langle Q_{\mathrm{k}}\right\rangle /\left\langle Q_{\mathrm{m}}\right\rangle$. We know that the stress level sets the total dissipation rate $\left(Q_{\mathrm{k}}+Q_{\mathrm{m}}\right)$ (e.g., Simon et al. 2009); stronger stresses extract more energy from the background shear flow, and that turbulence is rapidly dissipated into heat. However, does stronger turbulence by itself change the heating ratio, or is the change in the heating ratio mainly determined by changes in $P_{\mathrm{m}}$, which also increase the turbulence levels? This question of causality cannot be definitively answered from these data.

Further insight may come from examining the ratio of averaged Reynolds stress, $\left\langle\left\langle\rho v_{x} \delta v_{y}\right\rangle\right\rangle$, to averaged Maxwell stress, $\left\langle\left\langle-B_{x} B_{y}\right\rangle\right\rangle$, as a function of $\alpha$; this is shown in Fig. 9. The colors and symbols are the same as in Fig. 8 . The double brackets for the stresses denote time and volume averages, where the time average is calculated over the same 20 orbit period as in Fig. 8. There is a decrease in the ratio of the Reynolds to Maxwell stress as the total stress increases. These stresses are proportional to the perturbed magnetic and kinetic energies at the largest scales, and if this continued down to the dissipation scale, we might expect that the ratio $\left\langle Q_{\mathrm{k}}\right\rangle /\left\langle Q_{\mathrm{m}}\right\rangle$ would behave similarly with $\alpha$. In fact, the heating ratio shows the opposite trend with $\alpha$, indicating that a transfer of energy from magnetic to kinetic fluctuations must occur in the turbulent cascade.

Past net toroidal field simulations without explicit dissipation terms also find a trend for a decrease in the ratio of the Reynolds to Maxwell stress with increasing $\alpha$ (e.g., Hawley et al. 1995). So this may be a general result independent of $P_{\mathrm{m}}$. The quantity $\left\langle Q_{\mathrm{k}}\right\rangle /\left\langle Q_{\mathrm{m}}\right\rangle$ has not 
been extensively studied in past shearing box simulations, but Simon et al. (2009) found a ratio of $\sim 0.6$ for a net vertical field model without explicit dissipation terms.

In summary, these observations are consistent with the hypothesis that decreasing $P_{\mathrm{m}}$ increases the efficiency of magnetic reconnection and hence reduces the overall stress level. However, a more in-depth study would be required to better understand the full causal relationship between the ratio of dissipation terms and the saturation levels.

Finally, we note that the ratio of Reynolds stress to perturbed kinetic energy increases with increasing $\nu$, as shown in Fig. 10. There is no observed trend with $\eta$. As $\nu$ is increased, the fluid motions that are not being directly driven by the MRI become increasingly damped. The fluid motions that are driven by the magnetic field in the form of Reynolds stress follow the behavior of the Maxwell stress with $\nu$. This is also consistent with the hypothesis that increased $\nu$ leads to less efficient magnetic reconnection; the kinetic fluctuations become damped relative to the driving via the MRI, making it difficult to bring field lines close together for reconnection.

Overall, resistivity seems to play a more fundamental role than viscosity in these net toroidal field simulations. There is a critical $R m$ below which turbulence decays or fails to grow from linear perturbations. For a given resistivity near this critical value, a relatively low viscosity leads to MRI growth (linear regime) or sustained turbulence (nonlinear regime). A high viscosity can prevent growth (linear regime) or cause decay (nonlinear regime). Once the resistivity is sufficiently low to ensure MRI growth to saturation and continued turbulence, the effect of viscosity changes and higher viscosity gives larger $\alpha$ values.

\section{Discussion and Conclusions}

In this study, we carried out a series of local, unstratified shearing box simulations of the MRI with Athena including the effects of constant shear viscosity and Ohmic resistivity. The first simulations were initialized with a zero net magnetic flux in the domain for comparison with the results of F07. The second set of simulations are the first investigation of the impact of both viscosity and resistivity on models with a net toroidal field.

For the values of viscosity and resistivity they studied, F07 found that turbulence was sustained only above a critical $P_{\mathrm{m}}$ value, specifically when $P_{\mathrm{m}} \gtrsim 1$. There was evidence that this critical $P_{\mathrm{m}}$ value decreases as $R e$ increases (viscosity is reduced). We repeated these experiments and found that the saturation level of the MRI depends strongly on both viscosity and resistivity, and for every $R e$, there exists a critical $P_{\mathrm{m}}$ value below which the turbulence dies out. For those simulations where turbulence was sustained, we found good 
agreement between the Athena $\alpha$ values and those of F07.

Zero net field simulations are fundamentally different from net field models because an imposed background field cannot be removed as a result of the simulation evolution. The net field remains unstable and can drive fluid motion even during the fully nonlinear turbulence phase, assuming that that field was unstable to begin with. Lesur \& Longaretti (2007) examined the effects of diffusion on models with a net $\beta=100$ vertical field in a $1 \times 4 \times 1$ shearing box using a pseudo-spectral incompressible code. They found a relation $\alpha \propto P_{\mathrm{m}}^{\delta}$ with $\delta=0.25-0.5$ for values of $P_{\mathrm{m}}$ ranging from 0.12 to 8 , but they found no case where the turbulence died out completely for the range of viscosities and resistivities studied.

Among net field models, the purely vertical field case is significantly different from the purely toroidal field model, hence the need for the study we have presented here. For a vertical field, the linear MRI favors wavenumbers $k_{z} \sim \Omega / v_{\mathrm{A}}$ and $k_{x}=k_{y}=0$. The purely toroidal case favors $k_{y} \sim \Omega / v_{\mathrm{A}}$ with $k / k_{z}$ minimized. Since $k_{x}$ is time dependent due to the background shear, a given mode undergoes a finite period of amplification as $k_{x}$ swings from leading to trailing. These properties suggest that purely toroidal field models might be more sensitive to dissipation than the vertical field case.

In our numerical study of the linear growth regime of the toroidal MRI, we have found that increasing either the viscosity or the resistivity can prevent the growth of MRI modes. As the viscosity (resistivity) increases, the MRI needs a smaller resistivity (viscosity) in order to grow. However, for large enough values of either the viscosity or the resistivity, MRI growth is suppressed, even in the absence of the other dissipation term. Because of the importance of small wavelength (large wavenumber) modes, the critical $R m$ values, below which growth is inhibited, tends to be larger than what one would expect from an axisymmetric vertical field analysis, even in the absence of viscosity. Here, for comparable values of viscosity and resistivity, the critical $R m$ value was around 3200-6400.

Because the linear toroidal field MRI is time dependent, turbulence can only be sustained if nonlinear amplitudes are reached during the growth phase. Thus, the outcome of the linear MRI phase can be sensitive to the initial amplitude of the perturbations in a simulation where the viscous or resistive values are near the critical value.

In the nonlinear regime, we found that viscosity generally acts in an opposite sense to that in the linear regime; increased viscosity enhances angular momentum transport. Furthermore, increasing the resistivity appears to decrease the saturation level, in agreement with previous studies, and the critical $R m$, below which the turbulence dies is $\sim 800-1600$. Near the critical $R m$, however, increasing the viscosity causes the turbulence to decay, a behavior more in line with the linear regime. 
In our simulations, as well as those of Lesur \& Longaretti (2007), $P_{\mathrm{m}}<1$ did not necessarily quench the nonlinear turbulence or prevent growth from linear perturbations. Resistivity or viscosity above a certain level can stabilize the system against these perturbations, but if both are sufficiently small, their ratio has no influence on MRI growth. The presence of turbulence, however, is distinct from the saturation level of that turbulence, and here $P_{\mathrm{m}}$ can have a significant effect. For both net toroidal and net vertical field simulations, $\alpha$ increases with increasing $P_{\mathrm{m}}$ for the range of values studied.

What do these results imply for the effect of resistivity and viscosity on the MRI and on astrophysical systems? In principle, they could be quite significant. In protostellar disks, the resistivity is thought to be quite high near the midplane, leading to the existence of the dead zone (Gammie 1996). The $R m$ values studied here could be applicable to such systems. However, the implications for accretion disks with small values of viscosity and resistivity (e.g., X-ray binary disks) are less clear. Because the range of $\alpha$ values we obtained decreases with increasing $R e$ (Fig. [6), it is possible that $\alpha$ may converge to a single value independent of $P_{\mathrm{m}}$ as $R e$ and $R m$ are increased. If true, this would suggest that the dissipation scales might have very little influence on the saturation level of the MRI in astrophysical disks. This idea will need to be tested with higher resolution simulations to ensure that the (small) viscous and resistive scales are adequately resolved. If, on the other hand, $P_{\mathrm{m}}$ still has an influence on the turbulence even for very small values of viscosity and resistivity, our results (taken together with those in the literature) could be applicable to such disks. The resistivity, viscosity, and $P_{\mathrm{m}}$ can vary quite substantially in these systems, not only between different astrophysical objects, but also within a given disk (e.g., Brandenburg \& Subramanian 2005). Balbus \& Henri (2008) analyze a possible $P_{\mathrm{m}}$ dependence on radius in X-ray binaries to suggest that such a dependence could be at the core of spectral state transitions in these systems.

The work to date is suggestive, but there remain several limitations associated with these shearing box simulations. First, the simulations are unstratified; vertical gravity may also play a role in establishing the overall turbulent state. For example, Davis et al. (2009) carried out a series of zero net field shearing box simulations with vertical gravity and explicit dissipation and found that the turbulence does not decay as readily as in the unstratified case. Secondly, all of the simulations to date have explored a relatively restricted range of parameters. Here, for example, we have examined only one value for the toroidal field strength and one domain size. Finally, as touched upon above, the range of values for $R e$ and $R m$ that have been studied are somewhat restricted and certainly much smaller than would be appropriate for many astrophysical disk systems. While this limitation is partially computational and can be improved upon with higher resolutions, the question remains for astrophysical systems whether viscous and resistive processes that take place on relatively 
small lengthscales can have a significant influence on macroscopic stress terms whose scales are on order the pressure scale height in the disk. But regardless of the importance of resistivity and viscosity for astrophysical systems, the values of $R e$ and $R m$ are very important for setting $\alpha$ in numerical simulations, much more so than many other shearing box parameters (e.g., pressure) studied to date. Without a more thorough understanding of the role that dissipation terms play, quantitative predictions of $\alpha$ values from simulations will not be possible.

In summary, our experiments have explored the effect of changing viscosity and resistivity on MRI simulations with a net toroidal field. This work serves to expand upon previous investigations of the impact of small-scale dissipation. While the direct applicability of studies such as this to specific stress values within astrophysical systems remains uncertain, it is likely that for the conceivable future, numerical simulations will be our primary, if not only way to explore the nature of MRI-driven turbulence. A thorough understanding of MRI turbulence can only be obtained with a complete understanding of the effects of diffusion, both numerical and physical.

We thank Jim Stone, Steve Balbus, Jeremy Goodman, Pierre-Yves Longaretti, Martin

Pessah, Xiaoyue Guan, and Sebastien Fromang for useful discussions and suggestions regarding this work. We also thank the anonymous referee whose comments and suggestions improved this paper. This work was supported by NASA Headquarters under the NASA Earth and Space Science Fellowship Program Grant NNX08AX06H, a Virginia Space Grant Consortium fellowship, NASA grant NNX09AD14G, and NSF grant AST-0908869. The simulations were run on the TeraGrid Ranger system at TACC, supported by the National Science Foundation.

\section{REFERENCES}

Balbus, S. A., \& Hawley, J. F. 1991, ApJ, 376, 214

-. 1992, ApJ, 400, 610

—. 1998, Reviews of Modern Physics, 70, 1

Balbus, S. A., \& Henri, P. 2008, ApJ, 674, 408

Blackman, E. G., Penna, R. F., \& Varnière, P. 2008, New Astronomy, 13, 244

Brandenburg, A., \& Subramanian, K. 2005, Phys. Rep., 417, 1 
Colella, P. 1990, Journal of Computational Physics, 87, 171

Colella, P., \& Woodward, P. R. 1984, Journal of Computational Physics, 54, 174

Davis, S. W., Stone, J. M., \& Pessah, M. E. 2009, arXiv:0909.1570

Evans, C. R., \& Hawley, J. F. 1988, ApJ, 332, 659

Fleming, T. P., Stone, J. M., \& Hawley, J. F. 2000, ApJ, 530, 464

Fromang, S., \& Papaloizou, J. 2007, A\&A, 476, 1113

Fromang, S., Papaloizou, J., Lesur, G., \& Heinemann, T. 2007, A\&A, 476, 1123

Gammie, C. F. 1996, ApJ, 457, 355

Gardiner, T. A., \& Stone, J. M. 2005a, in AIP Conference Series, Vol. 784, Magnetic Fields in the Universe: From Laboratory and Stars to Primordial Structures., ed. E. M. de Gouveia dal Pino, G. Lugones, \& A. Lazarian (Melville, NY: AIP), 475-488

—. 2005b, Journal of Computational Physics, 205, 509

—. 2008, Journal of Computational Physics, 227, 4123

Guan, X., Gammie, C. F., Simon, J. B., \& Johnson, B. M. 2009, ApJ, 694, 1010

Hawley, J. F., Gammie, C. F., \& Balbus, S. A. 1995, ApJ, 440, 742

-. 1996, ApJ, 464, 690

Komissarov, S. S. 2007, MNRAS, 382, 995

Landau, L. D., \& Lifshitz, E. M. 1959, Fluid mechanics, Course of theoretical physics (Oxford: Pergamon Press)

Lesur, G., \& Longaretti, P.-Y. 2007, MNRAS, 378, 1471

Masada, Y., \& Sano, T. 2008, ApJ, 689, 1234

Papaloizou, J. C. B., \& Terquem, C. 1997, MNRAS, 287, 771

Pessah, M. E., Chan, C.-k., \& Psaltis, D. 2007, ApJ, 668, L51

Pessah, M. E., \& Chan, C.-k. 2008, ApJ, 684, 498

Sano, T., \& Inutsuka, S.-I. 2001, ApJ, 561, L179 
Sano, T., Inutsuka, S.-I., \& Miyama, S. M. 1998, ApJ, 506, L57

Sano, T., Inutsuka, S.-I., Turner, N. J., \& Stone, J. M. 2004, ApJ, 605, 321

Sano, T., \& Stone, J. M. 2002, ApJ, 577, 534

Simon, J. B., Hawley, J. F., \& Beckwith, K. 2009, ApJ, 690, 974

Stone, J. M., Gardiner, T. A., Teuben, P., Hawley, J. F., \& Simon, J. B. 2008, ApJS, 178, 137

Ziegler, U., \& Rüdiger, G. 2001, A\&A, 378, 668 


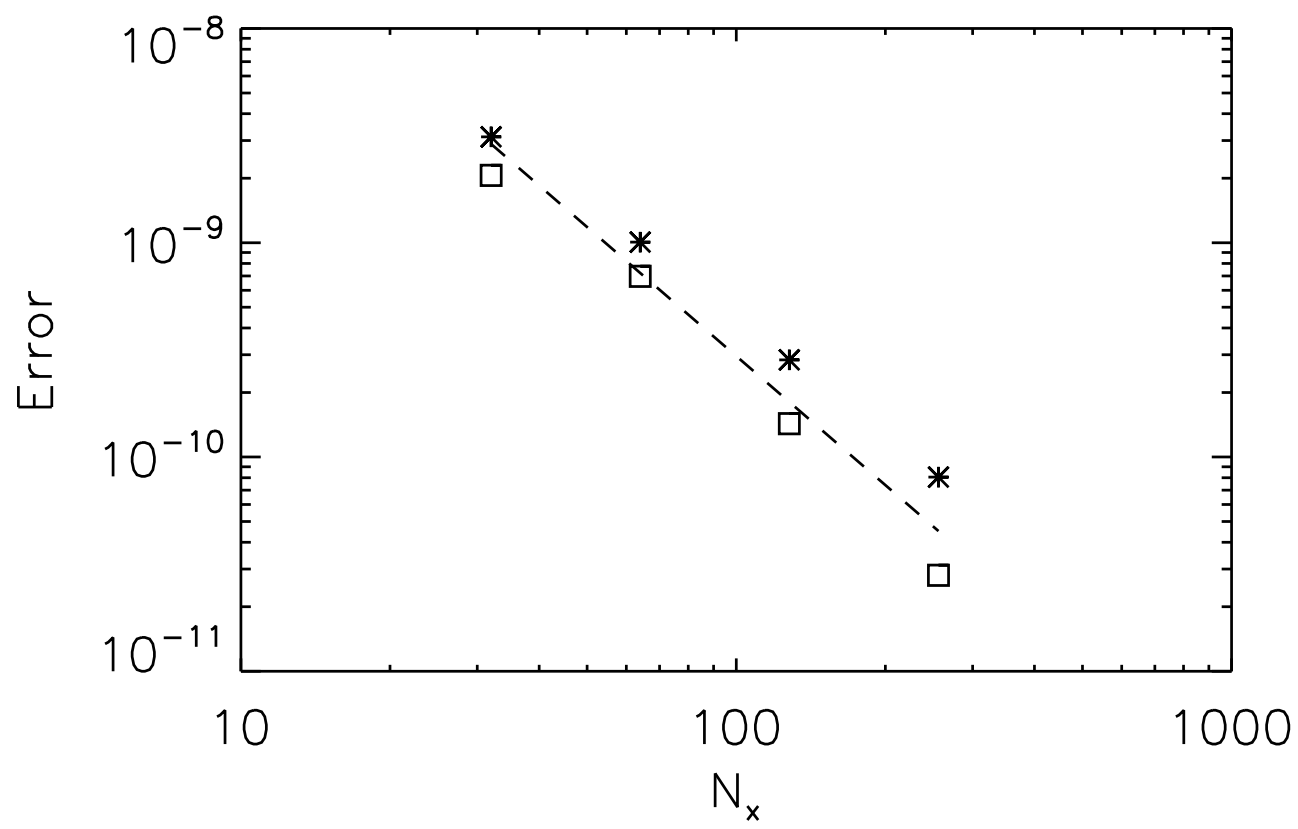

Fig. 1.- Numerical error as a function of $x$ resolution for the three-dimensional decaying linear wave problem. The boxes are the errors for a decaying Alfvén wave, and the asterisks are the errors for a decaying sound wave. The error is calculated from the square root of the sum of the squared errors in the density and momenta (for the sound wave) and the density, momenta, and magnetic field (for the Alfvén wave) obtained using the analytic solution. The dashed line shows the slope corresponding to second-order convergence. 


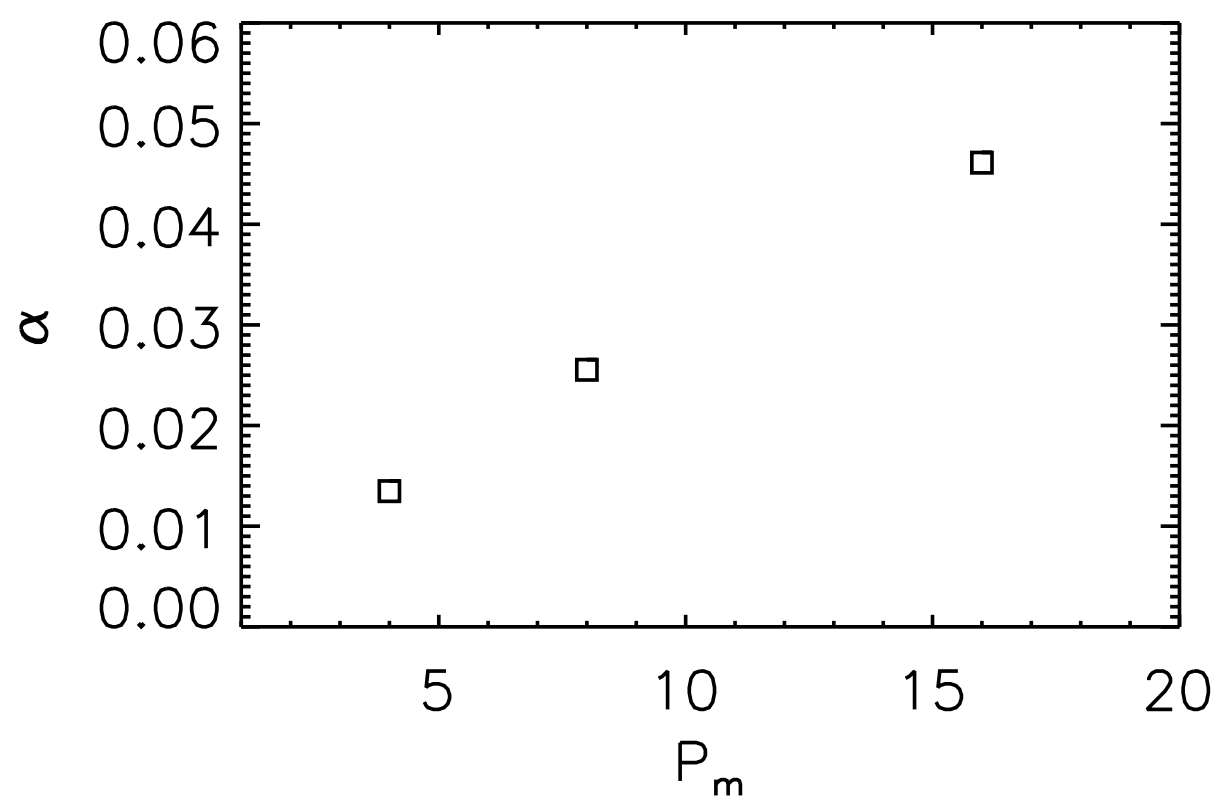

Fig. 2.- Time- and volume-averaged stress parameter $\alpha$ as a function of $P_{\mathrm{m}}$ in the SZ simulations; $\alpha \equiv\left\langle\left\langle\rho v_{x} \delta v_{y}-B_{x} B_{y}\right\rangle\right\rangle / P_{o}$, where the average is calculated over the entire simulation domain and from 20 orbits to the end of the simulation. Only simulations with sustained turbulence are plotted. The $P_{\mathrm{m}}=4$ model has $R m=12500$ whereas the other two have $R m=12800$. There is a nearly linear increase in $\alpha$ with $P_{\mathrm{m}}$. 


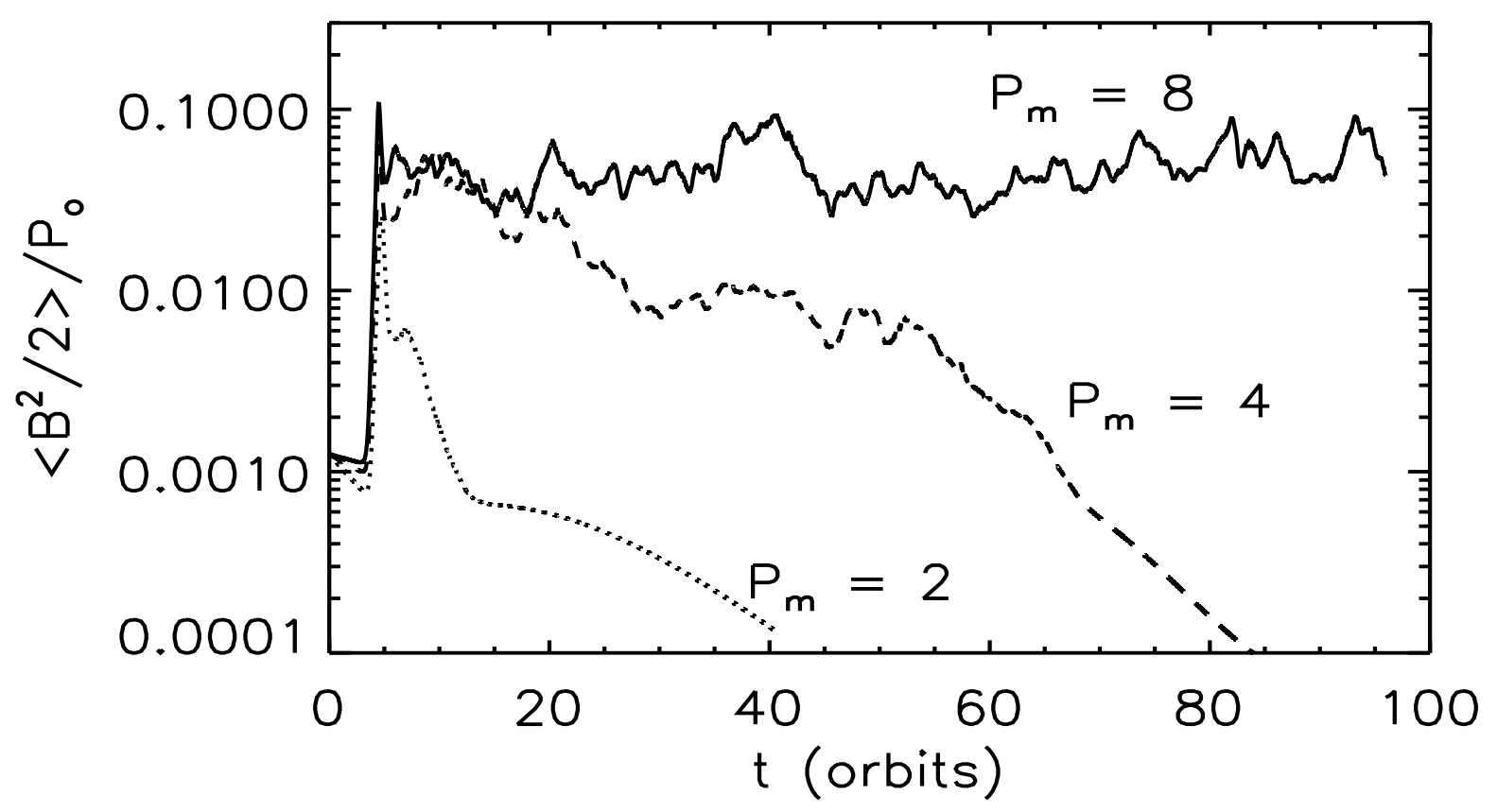

Fig. 3.- Time evolution of volume-averaged magnetic energy density normalized by the gas pressure for the SZ runs with $R e=1600$ and varying $P_{\mathrm{m}}$. The volume average is calculated over the entire simulation domain. The solid line corresponds to $P_{\mathrm{m}}=8$, the dashed line corresponds to $P_{\mathrm{m}}=4$, and the dotted line corresponds to $P_{\mathrm{m}}=2$. The turbulence decays for the lowest two $P_{\mathrm{m}}$ values, with the $P_{\mathrm{m}}=4$ case taking roughly 60 orbits to decay. 


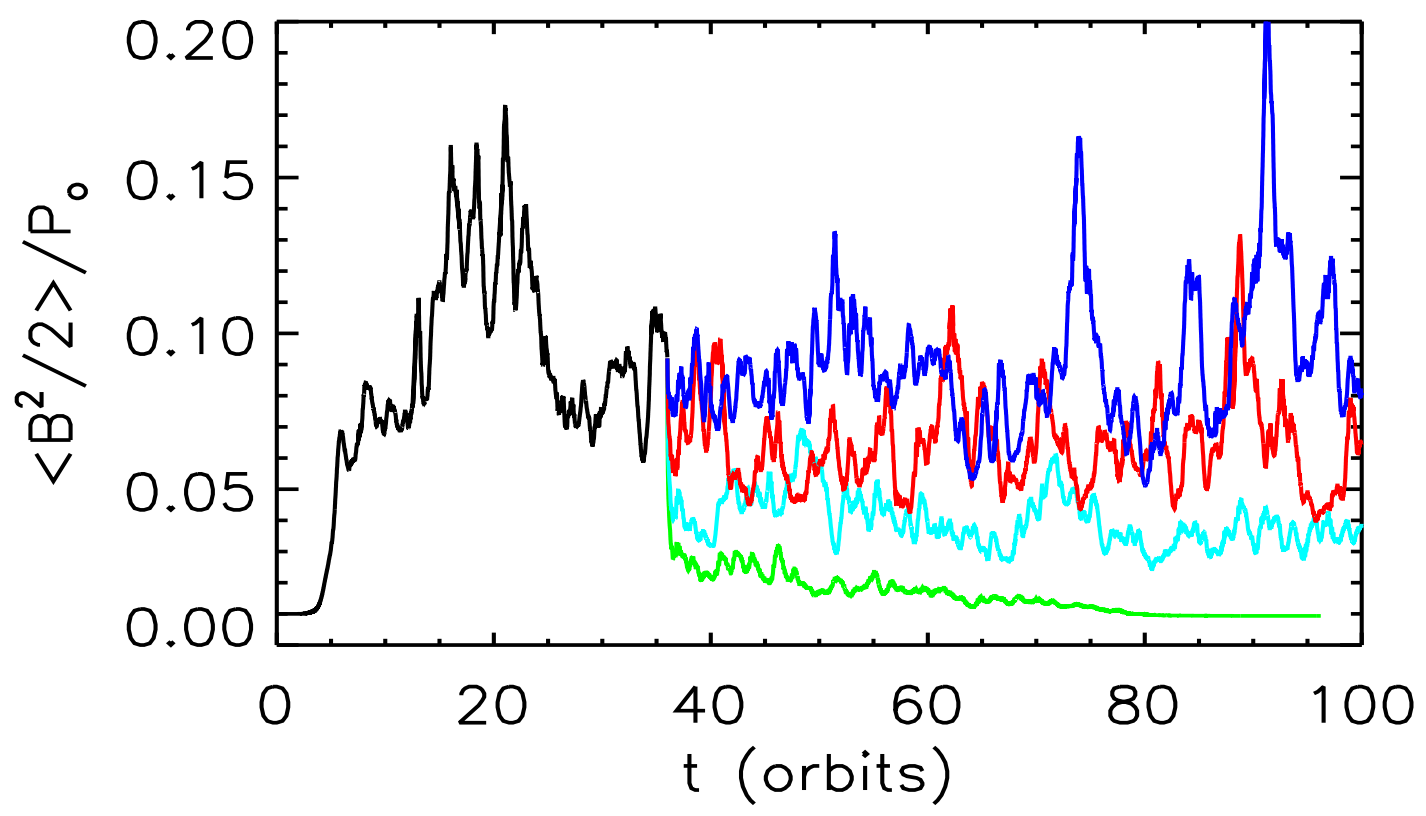

Fig. 4. - Time evolution of volume-averaged magnetic energy density normalized by the gas pressure for the YN runs with $R e=25600$ (black curve) and $R e=1600$ (colored curves). The volume average is calculated over the entire simulation domain. The colors indicate $P_{\mathrm{m}}$; green corresponds to $R m=800\left(P_{\mathrm{m}}=0.5\right)$, light blue to $R m=1600\left(P_{\mathrm{m}}=1\right)$, red to $R m=3200\left(P_{\mathrm{m}}=2\right)$, and dark blue to $R m=6400\left(P_{\mathrm{m}}=4\right)$. Increasing $R m\left(P_{\mathrm{m}}\right)$ leads to enhanced turbulence. 


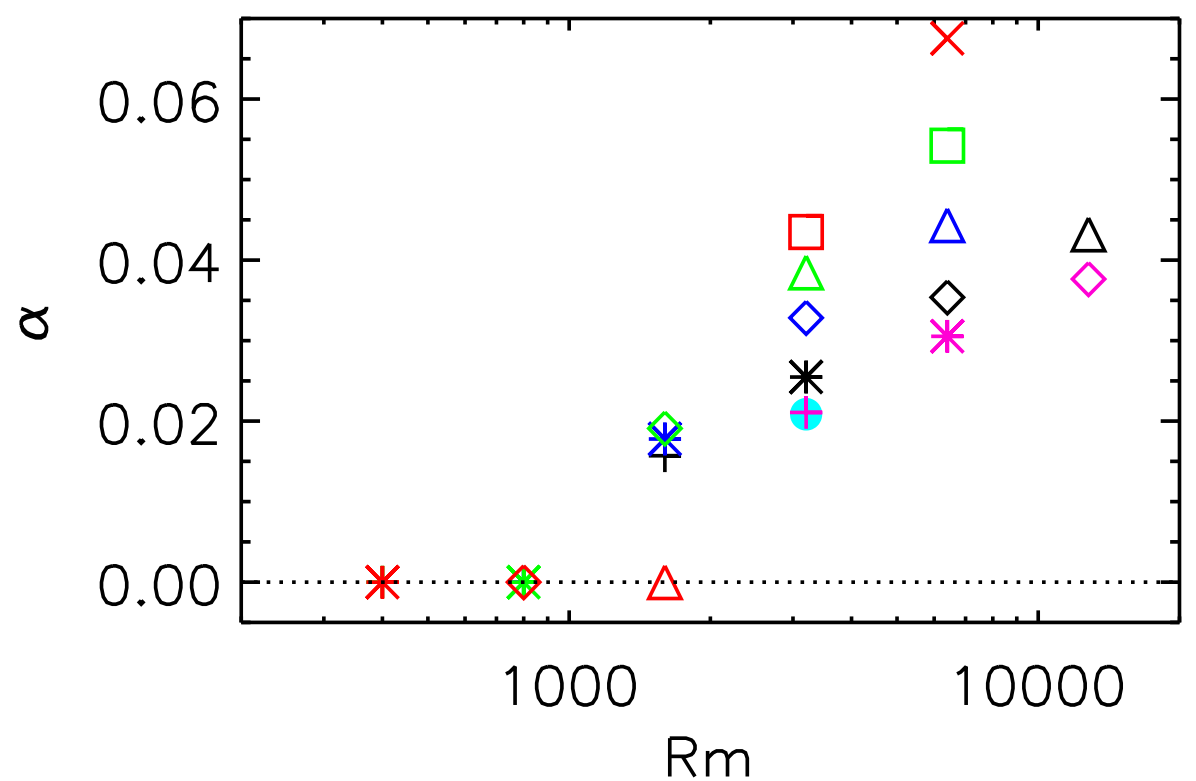

Fig. 5.- Time- and volume-averaged stress parameter $\alpha$ as a function of $R m$ in the YN simulations; $\alpha \equiv\left\langle\left\langle\rho v_{x} \delta v_{y}-B_{x} B_{y}\right\rangle\right\rangle / P_{o}$. The time average runs from 50 orbits onward, and the volume average is calculated over the entire simulation domain. The colors correspond to $R e$ values, and the symbols correspond to $P_{\mathrm{m}}$ values. Red symbols are $R e=400$, green $R e=800$, dark blue $R e=1600$, black $R e=3200$, pink $R e=6400$, and light blue are $R e=12800$. Circles are $P_{\mathrm{m}}=0.25$, crosses $P_{\mathrm{m}}=0.5$, asterisks $P_{\mathrm{m}}=1$, diamonds $P_{\mathrm{m}}=2$, triangles $P_{\mathrm{m}}=4$, squares $P_{\mathrm{m}}=8$, and X's are $P_{\mathrm{m}}=16$. Note that some of the decayed turbulence $(\alpha=0)$ simulations are not plotted for clarity. Increasing $R m$ results in larger $\alpha$ values, and for $R m$ less than 800-1600, the turbulence decays. 


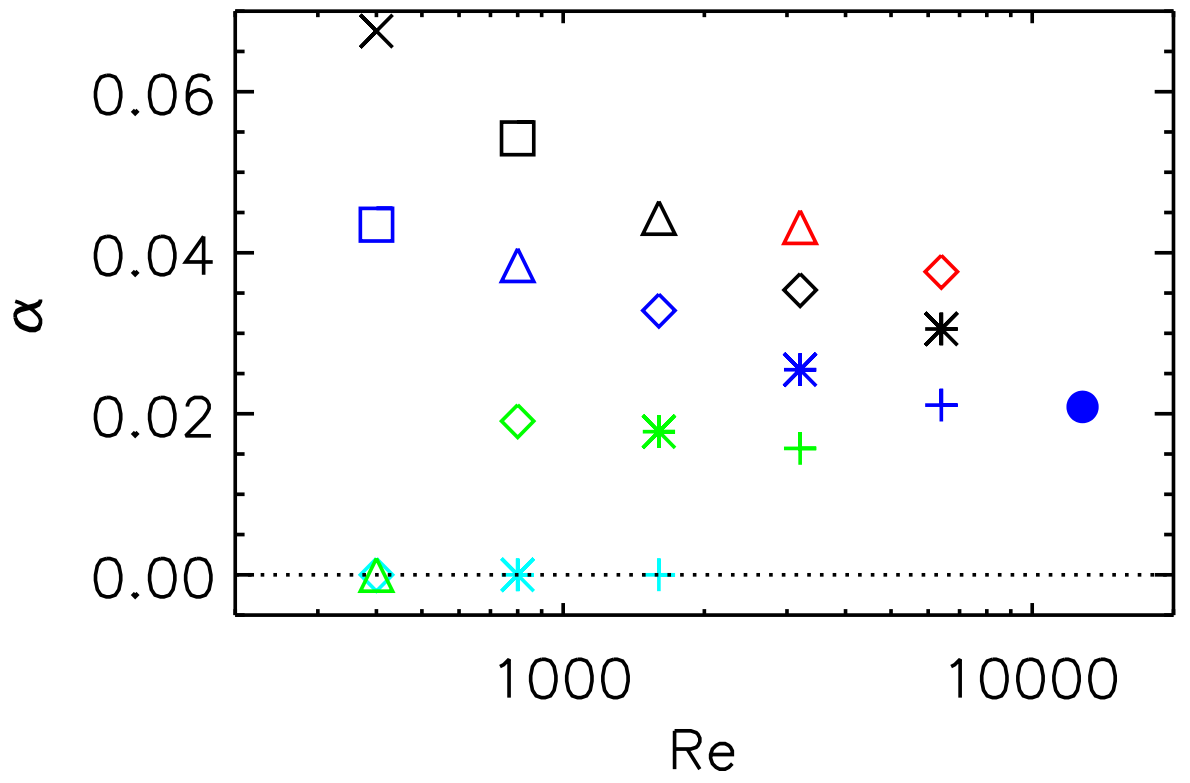

Fig. 6. - Time- and volume-averaged stress parameter $\alpha$ as a function of $R e$ in the $\mathrm{YN}$ simulations; $\alpha \equiv\left\langle\left\langle\rho v_{x} \delta v_{y}-B_{x} B_{y}\right\rangle\right\rangle / P_{o}$. The time average runs from 50 orbits onward, and the volume average is calculated over the entire simulation domain. The colors correspond to $R m$ values, and the symbols correspond to $P_{\mathrm{m}}$ values. Light blue symbols are $R m=800$, green $R m=1600$, dark blue $R m=3200$, black $R m=6400$, and red are $R m=12800$. Circles are $P_{\mathrm{m}}=0.25$, crosses $P_{\mathrm{m}}=0.5$, asterisks $P_{\mathrm{m}}=1$, diamonds $P_{\mathrm{m}}=2$, triangles $P_{\mathrm{m}}=4$, squares $P_{\mathrm{m}}=8$, and X's are $P_{\mathrm{m}}=16$. Note that some of the decayed turbulence $(\alpha=0)$ simulations are not plotted for clarity. Increasing $R e$ leads to decreasing $\alpha$ values. 


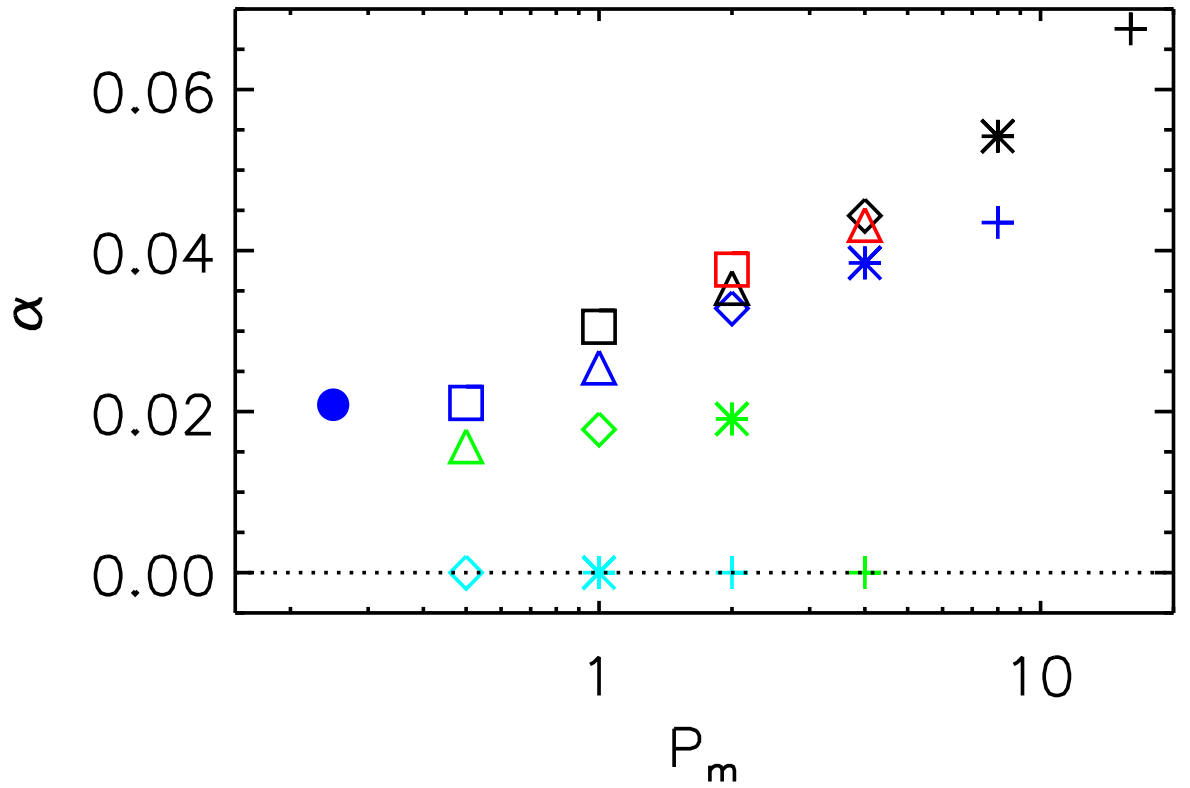

Fig. 7.- Time- and volume-averaged stress parameter $\alpha$ as a function of $P_{\mathrm{m}} ; \alpha \equiv$ $\left\langle\left\langle\rho v_{x} \delta v_{y}-B_{x} B_{y}\right\rangle\right\rangle / P_{o}$. The time average runs from 50 orbits onward, and the volume average is calculated over the entire simulation domain. The colors correspond to $R m$ values, and the symbols to $R e$ values. Light blue symbols are $R m=800$, green $R m=1600$, dark blue $R m=3200$, black $R m=6400$, and red are $R m=12800$. Crosses are $R e=400$, asterisks $R e=800$, diamonds $R e=1600$, triangles $R e=3200$, squares $R e=6400$, and circles are $R e=12800$. Note that some of the decayed turbulence $(\alpha=0)$ simulations are not plotted for clarity. The average stress increases with increasing $P_{\mathrm{m}}$. 

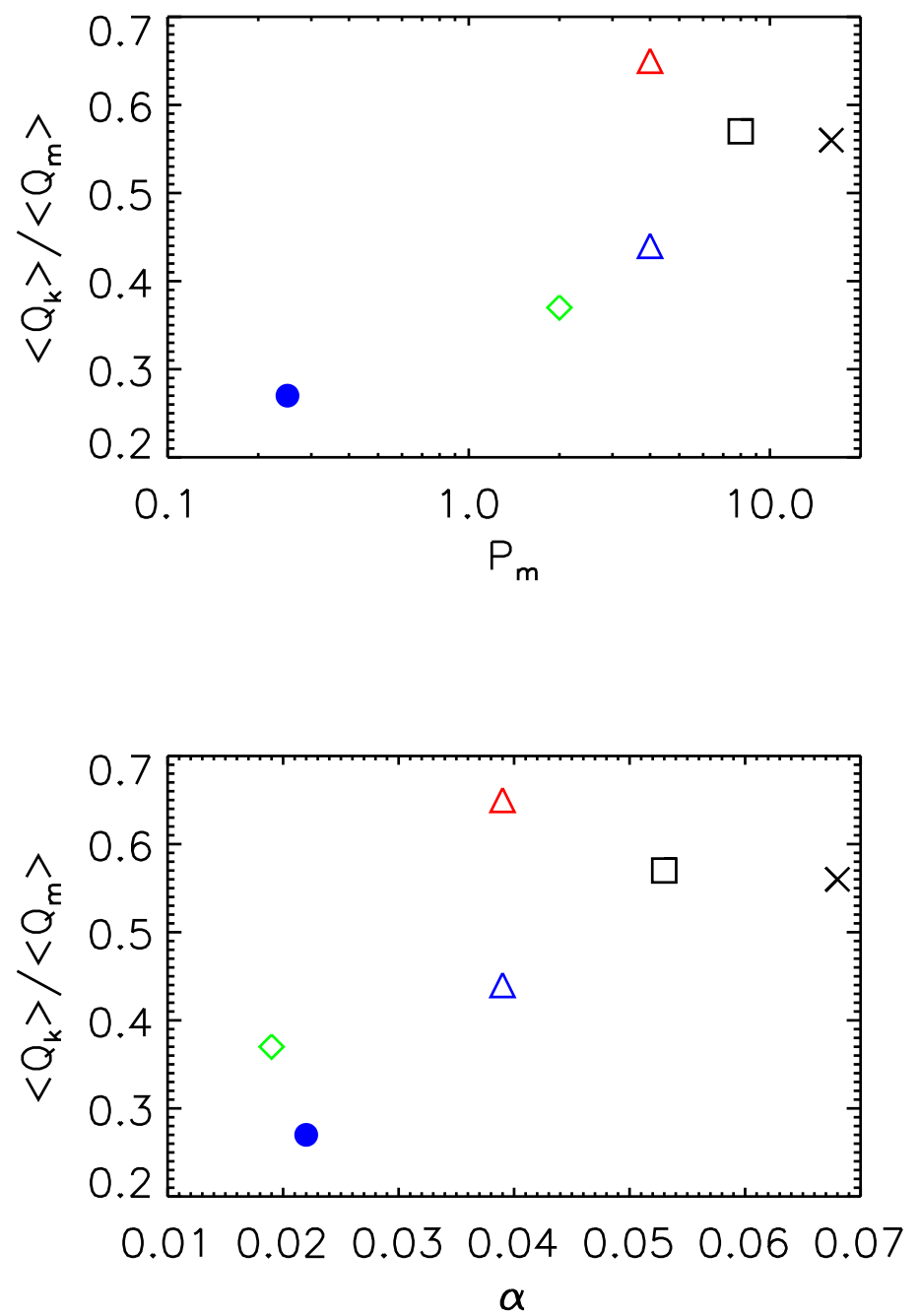

Fig. 8.- Ratio of kinetic to magnetic energy dissipation rate as a function of $P_{\mathrm{m}}$ (top panel) and $\alpha$ (bottom panel) for select YN runs; $\alpha \equiv\left\langle\left\langle\rho v_{x} \delta v_{y}-B_{x} B_{y}\right\rangle\right\rangle / P_{o}$. The colors and symbols are the same as in Fig. 6. The kinetic and magnetic dissipation rates as well as $\alpha$ have been averaged in volume and time. The volume average is calculated over the entire simulation domain and the time average is calculated from $t=70-90$ orbits for YNRe400Pm16 (black $\mathrm{X}$ ) and YNRe12800Pm0.25 (blue circle), $t=110-130$ orbits for YNRe800Pm2 (green diamond) and YNRe800Pm8 (black square), and $t=110.6-130.6$ orbits for YNRe800Pm4 (blue triangle) and YNRe3200Pm4 (red triangle). The ratio of viscous to resistive heating generally increases as either $\alpha$ or $P_{\mathrm{m}}$ increases, although not monotonically. 


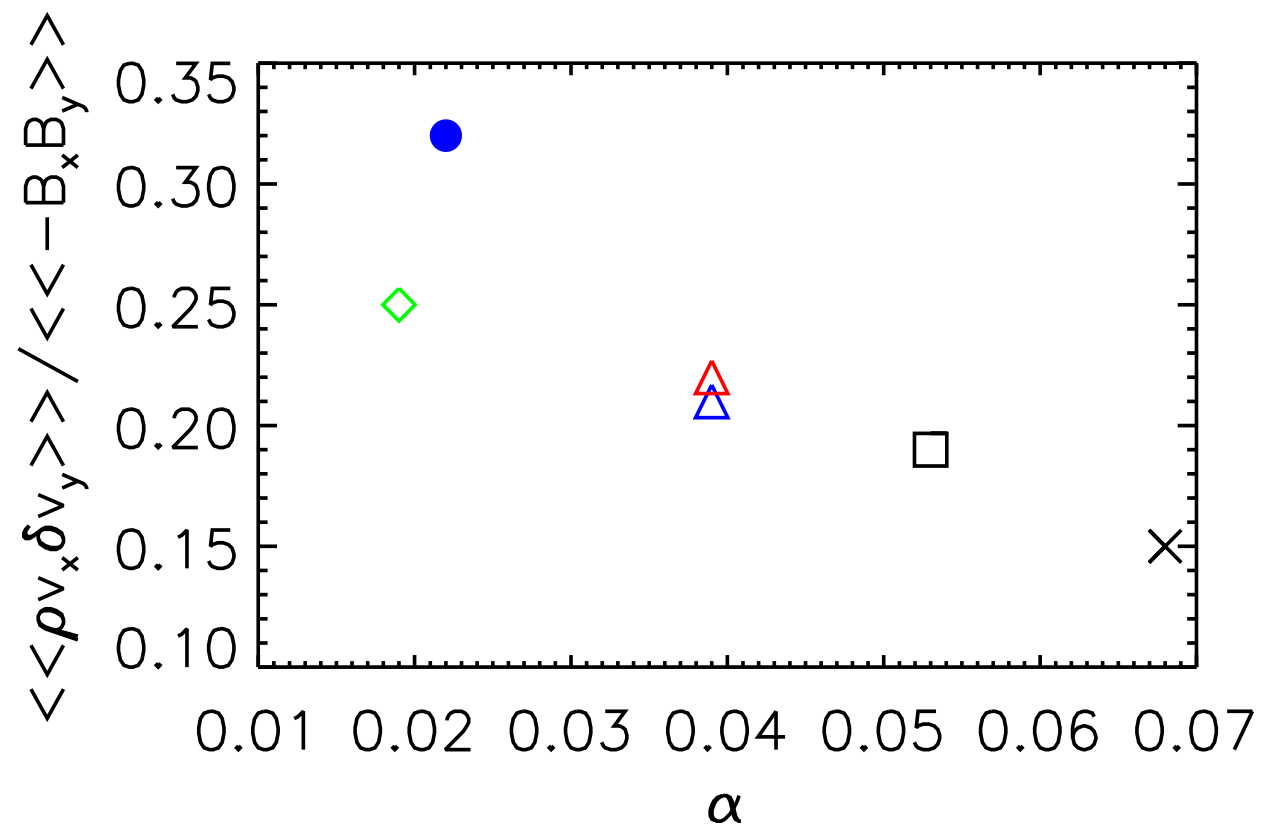

Fig. 9.- Ratio of Reynolds stress to Maxwell stress as a function of $\alpha$ for select YN runs; $\alpha \equiv\left\langle\left\langle\rho v_{x} \delta v_{y}-B_{x} B_{y}\right\rangle\right\rangle / P_{o}$. The colors and symbols are the same as in Fig. 6. The Maxwell and Reynolds stresses as well as $\alpha$ have been averaged in volume and time. The volume average is calculated over the entire simulation domain and the time average is calculated from $t=70-90$ orbits for YNRe400Pm16 (black X) and YNRe12800Pm0.25 (blue circle), $t=110-130$ orbits for YNRe800Pm2 (green diamond) and YNRe800Pm8 (black square), and $t=110.6-130.6$ orbits for YNRe800Pm4 (blue triangle) and YNRe3200Pm4 (red triangle). The ratio of Reynolds to Maxwell stress generally decreases with increasing $\alpha$. 


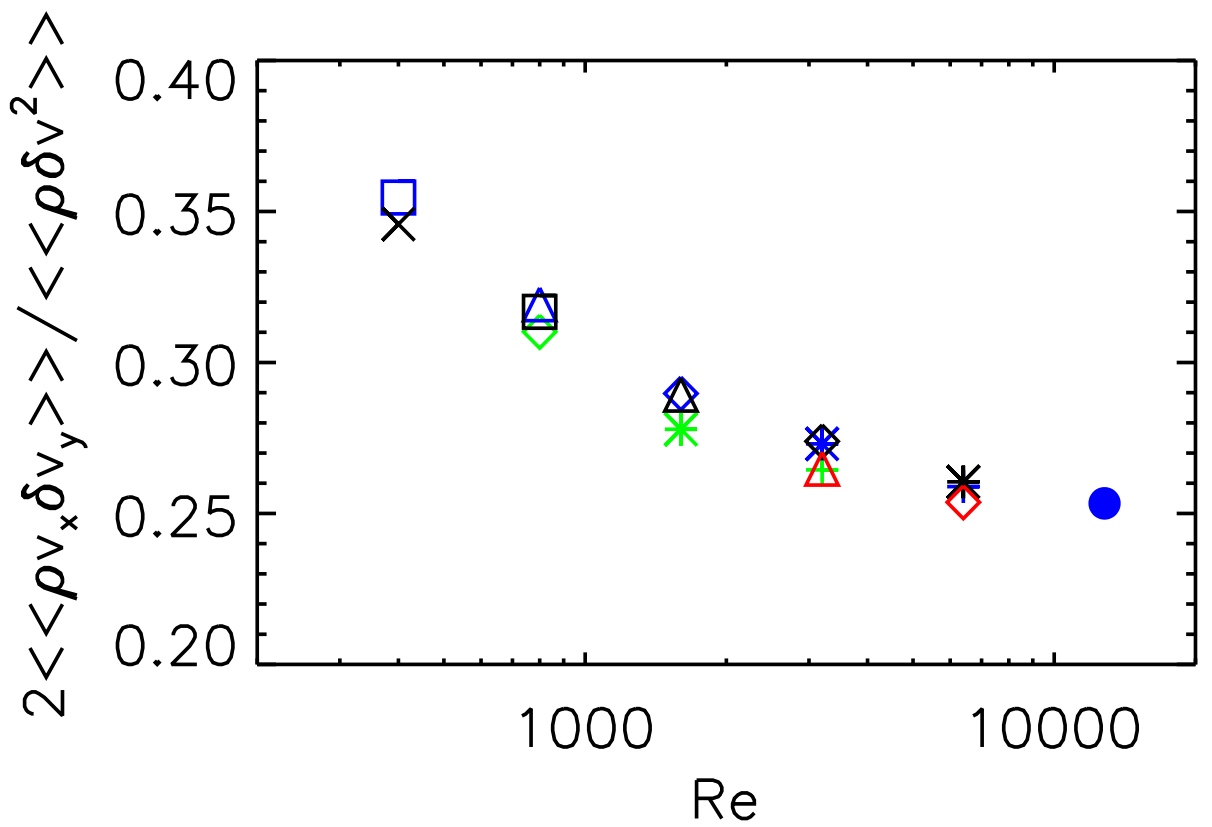

Fig. 10.- Ratio of Reynolds stress to perturbed kinetic energy as a function of Re in the sustained turbulence YN simulations. Both the Reynolds stress and the perturbed kinetic energy are time and volume averaged, with the time average calculated from orbit 50 onward and the volume average calculated over the entire simulation domain. The colors correspond to $R m$ values, and the symbols correspond to $P_{\mathrm{m}}$ values. Green symbols are $R m=1600$, blue $R m=3200$, black $R m=6400$, and red are $R m=12800$. Circles are $P_{\mathrm{m}}=0.25$, crosses $P_{\mathrm{m}}=0.5$, asterisks $P_{\mathrm{m}}=1$, diamonds $P_{\mathrm{m}}=2$, triangles $P_{\mathrm{m}}=4$, squares $P_{\mathrm{m}}=8$, and X's are $P_{\mathrm{m}}=16$. The ratio of stress to energy increases with increasing viscosity but does not change with resistivity. 